


\section{INSTITUTE FOR FUSION STUDIES}

DOE/ET-53088-455

IFSR \#455

Nonlinear Saturation of Ideal Interchange Modes in a Sheared Magnetic Field

A.D. BEKLEMISHEV*

Institute for Fusion Studies

The University of Texas at Austin

Austin, Texas 78712

* Permanent address: Kurchatov Institute of Atomic Energy, Moscow, U.S.S.R.

September 1990

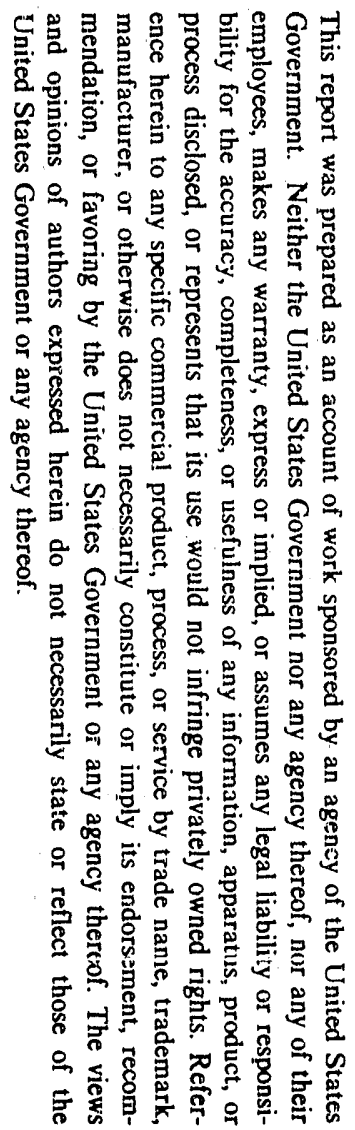

\section{THE UNIVERSITY OF TEXAS}

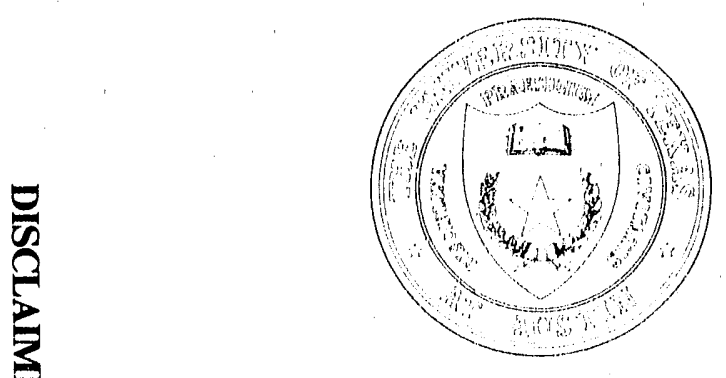

Recsived by OSTI

OCT 091990

AUSTIN

$\mathrm{DOE} / \mathrm{ET} / 53088--455$

DE91 000575 
September 1990

\title{
Nonlinear Saturation of Ideal Interchange Modes In a Sheared Magnetic Field
}

\author{
A. D. Beklemishev ${ }^{a}$ \\ Institute for Fusion Studies \\ The University of Texas at Austin \\ Austin, Texas 78712
}

Pressure-driven ideal modes cannot completely interchange flux tubes of a sheared magnetic field; instead, they saturate, forming new helical equilibria. These equilibria are studied both analytically and numerically with reduced MHD equations in a flux-conserving Lagrangian representation. For unstable localized modes, the structure of the nonlinear layer generated around the resonant flux surface depends on the value of Mercier parameter $D_{M}$. Its width is found to be proportional to the position of the inflection point on the linear eigenfunction. Perturbed surfaces in equilibrium always have folds, i.e., areas where the direction of the original reduced magnetic field is reversed. But only far from the instability threshold does the internal structure of the nonlinear layer resemble 'bubble' formation. The appearance of sheet currents and island-like structures along the resonant flux surface may be of interest for the description of forced reconnection in models with finite resistivity. Analytic results are found to be in agieement with 2-D numerical simulations. This study also includes the case of ballooning instability by representing nonlocal driving terms through the matching parameter $\Delta^{\prime}$, which defines the outer boundary conditions for the interchange layer.

\footnotetext{
a) Permanent address: I. V. Kurchatov Institute of Atomic Energy, Moscow, USSR 123182
} 


\section{Introduction}

The nonlinear behavior of ideal ballooning modes, which is a well-known problem in tokamak theory, is still a mystery even as the present generation of tokamak experiments is entering regimes for which the emergence of this instability has been predicted. The major difficulty is the use of the 'ballooning' transformation, developed for the representation of complex 3-D structures of linear eigenmodes, in the description of the nonlinear system. To avoid this difficulty, we first consider less complicated models, such as the localized interchange instability, and then try to extrapolate these results to more interesting situations.

If considered in a 'cylindrical tokamak' environment - i.e. in a straight cylindrical pinch with a strong parallel magnetic field, nonuniform current density, and periodicity requirements for any perturbation - the interchange instability reseribles the local behavior of ballooning modes in a tokamak. Both of them are driven by the unfavorable curvature of the magnetic field lines and the gradient of the plasma pressure and are stabilized by the shear of the magnetic field, which allows the perturbation to grow only in the vicinity. of those flux surfaces where the helical structure of the mode is resonant with the structure of the magnetic field. The main difference between interchange and ballooning modes is that in a ballooning perturbation, poloidal harmonics interact to utilize the modulated curvature of magnetic field lines in tokamak geometry, while the interchange mode is driven by the mean curvature on the flux surface. Since different poloidal harmonics have separate resonances with the magnetic field, the ballooning mode is much broader in radius and can be thought of as consisting of several radially shifted but partially overlapping perturbations with different helical structures. The interchange mode is only one such perturbation. Thus, once we have a sufficient understanding of its structure, it will be possible to consider toroidal corrections and the interaction of these modes.

For one ideal MHD instability there already is an accepted theory of nonlinear saturation. 
This is the $m=1$ internal kink mode in a 'cylindrical tokamak'. ${ }^{1-3}$ It was studied in real space fux-surface-based coordinate systems in order to find a new helical equilibrium. The problem was separated into a reduced description of the nonlinear layer around the resonance and a linearized description of the external perturbation. The amplitude of the saturated helical displacement was found from a matching procedure in which the driving terms from the external region were balanced by the formation of sheet currents along the resonant surface. In this paper we use a similar approach to study the interchange modes, and hence the equations and results are often comparable.

There are, of course, several qualitative differences between the nonlinear interchange mode and the internal kink mode. In particular, it is found that the internal structure of the nonlinear interchange layer is more complex than that of the $m=1$ mode, $^{1}$ and the usual ordering $\partial^{2} \psi / \partial r^{2} \gg \partial^{2} \psi / \partial \theta^{2}$ has only limited applicability. For large values of the local pressure gradient (described by the Mercier stability parameter $D_{M}$ ), the stabilizing influence of the magnetic field at the resonance is reduced al d the form of the flux surfaces in the new equilibrium resembles the formation of 'bubbles', predicted earlier for the external kink. ${ }^{4}$

The present study is based on the ideal MHD description for a plasma, with a high aspect ratio tokamak ordering for the equilibrium parameters. Throughout most of the paper, a simple 1-D geometry for the initial equilibrium and a 2 - $D$ description for the helical perturbations are assumed. This restricts us to single-helicity perturbations. The relationship between the 2-D localized interchange and 3-D ballooning modes is discussed only in Section VI. The present study is also limited to internal fixed-boundary perturbations and resorts to slab-like models whenever possible.

In Section II, the simplified equations for the descripcion of the nonlinear localized helical perturbation will be derived. These equations are a generalization of the layer equations of Ref.1 to accourit for local pressure gradients and the finite poloidal wavelength of the pertur- 
bation. Before presenting the solution we will discuss the general features of the system and the properties of the 'most interesting' solution in Sec. III and IV. In Sec. III an asymptotic method for the analytic evaluation of the radial width of the saturated state is presented. This width is relatively independent of the structure of the nonlinear layer, which is qualitatively described in Sec. IV with the hydrostatic model. Analytic estimates are found to be in agreement with the results of the numerical solution, presented in Sec. V. These numerical solutions are based on another form of the equations, derived in the Appendix, which form is necessary in order to avoid coordinate-related discontinuities during the description of folds or bubbles on flux suruces. The relationship between this problem and the full toroidal description of ideal ballooning modes is discussed in Sec. VI, and an estimate of the saturation level for the $\Delta^{\prime}$-driven layer is found. In Sec. VII, we summarize the established features of interchange-like modes and related unresolved problems.

\section{Simplified Equations}

Reduced MHD The localized interchange can be studied within the framework of reduced MHD, provided that curvature terms are retained to second order in the inverse aspect ratio during the derivation. Such a set of equations has been given by Drake and Antonsen ${ }^{5}$ :

$$
\frac{c^{2}}{B_{o}^{2}} \nabla_{\perp} \rho \frac{d}{d t} \nabla_{\perp} \phi=\vec{B} \cdot \nabla \frac{J_{\|}}{B}+2 c \frac{\vec{b} \times \vec{\kappa}}{B} \cdot \nabla p .
$$

Here $\vec{b} \times \vec{\kappa}=-\nabla \Phi \times \nabla R+\vec{b} \cdot \nabla\left(\nabla \psi /\left(R_{o} B_{0}\right)\right), \vec{B}=\nabla \Phi \times \nabla \psi+B_{o} R_{o} \nabla \Phi ; \Phi$ and $\psi$ are the normalized fluxes of magnetic field; $B_{\circ}$ is the magnetic field on magnetic axis, and $R_{\circ}$ is its radius; $\phi$ is the electrostatic potential, $p$ is the plasma pressure and $J_{\|}$is the density of parallel current; and $\vec{b}=\vec{B} / B$. The vorticity equation (1) has to be solved together with Ampere's and Ohm's laws,

$$
\begin{aligned}
R^{2} \nabla \cdot R^{-2} \nabla \psi & =\frac{4 \pi}{c} R_{o} B_{o}\left(J_{\|} / B\right), \\
\frac{1}{c R} \frac{\partial \psi}{\partial t} & =\vec{b} \cdot \nabla \phi
\end{aligned}
$$


which define the evolution of $J_{\|}$, and with the continuity equations that govern $p$ and $\rho$. For the latter we assume the convective model with

$$
\frac{d p}{d t}=0
$$

and $\rho=$ const. The transverse motion of the fluid, necessary for the description of inertia, is given by

$$
\vec{v}_{\perp}=-\frac{c R_{0}}{B_{0}} \nabla \phi \times \nabla \Phi+\frac{2 c \phi}{B} \vec{b} \times \vec{\kappa}
$$

Equation (1) can be simplified for a single-helicity perturbation in an axisymmetric cylindrical equilibrium. Following Ref. 4 we introduce the 'reduced field' $\vec{b}^{*}=\nabla z \times \nabla \psi^{*}$, and the 'reduced flux' $\psi^{*}$ as the variable part of the magnetic field $\vec{B}=B_{0}\left(\nabla z+\kappa \nabla z \times \nabla r+\vec{b}^{*}\right)$. Using homogeneity of the system along the helical axis of symmetry $\vec{\epsilon}_{\zeta} \cdot \nabla=0$, where $\vec{e}_{\zeta}=\nabla z+\kappa \nabla z \times \nabla r$, we obtain

$$
\frac{d}{d t} \Delta_{\perp} \phi=\left(b^{*} \cdot \nabla\right) \Delta_{\perp} \psi^{*}+\beta_{\psi}\left(b^{*} \cdot \nabla\right)\left(\nabla \psi^{*}\right)^{2}+2 \kappa^{2} \beta_{\psi} \frac{\partial \psi^{*}}{\partial \theta}
$$

In these terms Ohm's law can be expressed as a continuity equation for the reduced flux

$$
\frac{d \psi^{*}}{d t}=0
$$

which implies conservation of magnetic surfaces throughout all possible motions.

Because of the incompressibility assumption, the pressure gradient is a function of flux surface. It appears in Eq. (6) through $\beta_{\psi}(\psi)=4 \pi B_{o}^{-2}\left(\partial p_{o} / \partial \psi^{*}\right)$. Also, we have renormalized the electrostatic potential and discarded the variation of $\rho$.

Lagrangian Coordinates The plasma motion can be described as a mapping or a Lagrangean transformation from the initial position of the fluid element to its final position $\left(r_{o}, \theta_{o}\right) \rightarrow(r, \theta)$. Following Ref. 3, we rewrite the equations to define this transformation rather than that of the potentials. From the flux conservation law (7) we have $\psi^{*}=\psi_{0}\left(r_{0}\right)$, 
$\beta_{\psi}=\beta^{\prime}\left(r_{0}\right) / \psi_{0}^{\prime}\left(r_{0}\right)$, and incompressibility means that the Jacobian of the transformation is conserved:

$$
\left[r^{2}, \theta\right]=\left[r_{o}^{2}, \theta_{0}\right]=2 r_{o}
$$

Here $[a, b]$ is our notation for Poisson brackets:

$$
[a, b] \equiv \frac{\partial a}{\partial r_{o}} \frac{\partial b}{\partial \theta_{o}}-\frac{\partial a}{\partial \theta_{o}} \frac{\partial b}{\partial r_{o}} \equiv a_{r} b_{\theta}-a_{\theta} b_{r}
$$

where subscripts will be used to denote partial derivatives in $\left(r_{0}, \theta_{0}, t\right)$ space, whenever compactness is required.

Substituting the velocity of the fluid instead of the electrostatic potential (via Eq. (5)) into the left hand side of Eq. 6, and expressing gradients in terms of Poisson brackets, we obtain

$$
\begin{aligned}
\omega_{A}^{-2}\left(\left[r_{t t}, r\right]+\left[r^{2} \theta_{t}, \theta\right]_{t}\right)=\frac{\psi_{o}^{\prime}}{r_{o}} \frac{\partial}{\partial \theta_{o}}( & {\left.\left[r^{2} \frac{\psi_{o}^{\prime}}{r_{o}} \theta_{\theta}, \theta\right]+\left[\frac{\psi_{o}^{\prime}}{r_{o}} r_{\theta}, r\right]\right) } \\
& +\beta^{\prime}\left(\frac{\psi_{o}^{\prime}}{r_{o}}\right)^{2} \frac{\partial}{\partial \theta_{o}}\left(r^{2} \theta_{\theta}^{2}+r_{\theta}^{2}\right)+2 \beta^{\prime} \kappa^{2} r \frac{\partial r}{\partial \theta_{o}}
\end{aligned}
$$

Here $\omega_{A}=\kappa c_{A} / r$ is the normalization constant for the Alfvén frequency.

As shown in Ref. 3, an equation of this type has solutions with asymptotic behavior resembling the phase mixing of linear Alfvén waves. For low frequency oscillations, this. mixing is due to the radial dependence of $k_{\|}$in a sheared magnetic field. It provides an effective channel for the 'dissipation' of kinetic energy into short-wavelength fluctuations with the characteristic time $\tau_{A}=k_{\perp} /\left(c_{A} k_{\|}^{\prime}\right)$. This feature is important for understanding the role of the helical equilibrium. Once the axisymmetric equilibrinm becomes unstable, the plasma column will not oscillate around the stable helical one, as might be expected in an ideal system with few degrees of freedom. Kinetic energy will be lost in small-scale fluctuations and the column will asymptotically approach the helical configuration. Completion of this process, though, will require an infinite time, which in real physical system means the onset of reconnection. ${ }^{6}$ 
We now proceed by disregarding inertial terms in the left hand side of Eq. (10), so that the remainder describes equilibrium configurations only. It can be integrated in $\theta_{0}$ to yield

$$
\frac{\psi_{0}^{\prime}}{r_{0}}\left[r^{2} \frac{\psi_{0}^{\prime}}{r_{0}} \theta_{\theta}, \theta\right]+\frac{\psi_{0}^{\prime}}{r_{0}}\left[\frac{\psi_{o}^{\prime}}{r_{0}} r_{\theta}, r\right]+\beta^{\prime}\left(\frac{\psi_{0}^{\prime}}{r_{0}}\right)^{2}\left(r^{2} \theta_{\theta}^{2}+r_{\theta}^{2}\right)+\beta^{\prime} \kappa^{2}\left(r^{2}-r_{o}^{2}\right)=H\left(r_{0}\right) .
$$

Here $H\left(r_{o}\right)$ is the integration constant. Its value must be found from the boundary conditions in $\theta_{o}$, namely, the periodicity requirements. The derivative of the flux can be expressed in terms of the rotational transform of the magnetic field:

$$
\chi \equiv \psi_{o}^{\prime} / r_{o}=\mu\left(r_{o}\right)-\alpha
$$

where $\mu=1 / q$, and $\alpha=n / m$ is the helicity of the perturbation. We also note that the third term on the left is only a small correction, of the order of $\beta$, to the sum of the first and the second terms, and is not necessary in our approximation.

Thus, any mapping between two equilibria $\left(r_{o}, \theta_{0}\right) \rightarrow(r, \theta)$, which is consistent with flux conservation requirements, must satisfy

$$
\chi\left[r^{2} \chi \frac{\partial \theta}{\partial \theta_{0}}, \theta\right]+\chi\left[\chi \frac{\partial r}{\partial \theta_{0}}, r\right]+\beta^{\prime} \kappa^{2}\left(r^{2}-r_{o}^{2}\right)=H\left(r_{0}\right)
$$

Mixed Coordinates Up to this point we have a description of equilibrium based on two equations, (8) and (13), for two functions $r\left(r_{o}, \theta_{0}\right)$ and $\theta\left(r_{0}, \theta_{0}\right)$. It is possible to rewrite this problem as a single equation for one function by introducing the new mixed pair of independent variables: $\left(r_{0}, \theta\right)$. The first one is the initial radial position of the fluid element in an axisymmetric equilibrium, and hence labels magnetic surfaces, but the second one is the poloidal angle of the same element in the final helical equilibrium. For convenience we also define the displacements

$$
\xi\left(r_{o}, \theta\right)=r-r_{o}, \quad \lambda\left(r_{o}, \theta\right)=\theta-\theta_{o}
$$

and the following shorthand notations for derivatives:

$$
\hat{a}_{\theta} \equiv\left(\frac{\partial a}{\partial \theta}\right)_{r_{o}}, \quad \hat{a}_{r} \equiv\left(\frac{\partial a}{\partial r_{o}}\right)_{\theta} .
$$


In terms of these variables the incompressibility condition (8) reduces to

$$
1-\hat{\lambda}_{\theta}=\frac{r}{r_{o}}\left(1+\hat{\xi}_{r}\right)
$$

and $[a, b]_{r_{0}, \theta_{o}}=\left(1-\hat{\lambda}_{\theta}\right)^{-1}[a, b]_{r_{0}, \theta},\left(\partial / \partial \theta_{o}\right)_{r_{0}}=\left(1-\hat{\lambda}_{\theta}\right)^{-1}(\partial / \partial \theta)_{r_{o}}$. Using these identities it is possible to remove all references to $\theta_{0}$ and $\lambda$ from Eq. (13). We obtain

$$
\begin{aligned}
\left(\frac{1}{r} \frac{r_{o} \chi}{1+\hat{\xi}_{r}}\right) \frac{\partial}{\partial r_{o}}\left(r \frac{r_{o} \chi}{1+\hat{\xi}_{r}}\right)+\frac{1}{2} \frac{\partial}{\partial r_{o}}\left(\frac{r_{o}}{r} \frac{\chi \hat{\xi}_{\theta}}{1+\hat{\xi}_{r}}\right)^{2}- \\
-\frac{r_{o}}{r} \chi \frac{\partial}{\partial \theta}\left(\frac{r_{o}}{r} \frac{\chi \hat{\xi}_{\theta}}{1+\hat{\xi}_{r}}\right)+\beta^{\prime} \kappa^{2}\left(r^{2}-r_{o}^{2}\right)=H\left(r_{o}\right) .
\end{aligned}
$$

We are able to see the relationship of this equation with the linear theory if we multiply it by $r_{\circ}$ and linearize for $\xi \ll r_{0}$. Then it becomes

$$
\frac{\partial}{\partial r_{0}}\left(r^{3} \chi^{2} \frac{\partial \xi}{\partial r_{o}}\right)+r \chi^{2} \xi+r \chi^{2} \frac{\partial^{2} \xi}{\partial \theta^{2}}-2 r^{2} \beta^{\prime} \kappa^{2} \xi=0
$$

where $\chi^{2}=(\mu-n / m)^{2}$, and the first two terms originate from the first term of Eq. (1T). This equation is widely used for the description of kink and localized modes in the cylindrical approximation. ${ }^{8}$

Comparing this linearization with equation (17), we note that probably the most important nonlinearity is related to the $1+\hat{\xi}_{r}$ denominators. Its physical meaning is that for $\hat{\xi}_{r}=-1$, the flux surfaces touch one another at this point and a sheet current is. formed, which makes the energy of such perturbation infinite, if $\chi \neq 0$.

Radial Localization To proceed further, we have to make assumptions about the unknown solution. This procedure requires verification, which is provided in Sec. V by a comparison of the numerical solution of Eq. (13) with that of the simplified version derived below.

The usual assumption, applicable to linear localized modes or to the nonlinear layer of the $m=1$ internal kink mode, ${ }^{1}$ is that in the vicinity of the resonant surface $\chi=0$, the radial 
variation of perturbed quantities is much larger then the poloidal variation, $\partial^{2} \psi / \partial r^{2} \gg$ $r^{-2}\left(\partial^{2} \psi / \partial \theta^{2}\right)$. We use this inequality to introduce the ordering

$$
\left\{\begin{array}{l}
x \sim\left(r-r_{o}\right) / r \sim \epsilon \ll 1 \\
k_{\theta}=\partial / \partial \theta \sim \epsilon r \partial / \partial r \gg 1 \\
\epsilon \ll \kappa^{2} \beta^{\prime} r<1
\end{array}\right.
$$

With the above assumptions, the second and third terms in Eq. (17) contribute to force balance only outside of the $\chi \sim \epsilon$ nonlinear layer. It means that their linearized form can be used instead of the full expression and all the important nonlinearity is hidden in the first term. Indeed, this part of the equation is essentially the same as $\left(\nabla_{r}\right)^{2} w=0$, used for a layer description in Ref. 1.

The assumption $k_{\theta} \gg 1$, valid for localized modes, simplifies the cylindrical model into the slab-like one, since we can now discard variation of the metric with $r$ and expand all quantities around the point of resonance $r_{\chi}$, where $\chi\left(r_{\chi}\right)=0$ or $\mu\left(r_{\chi}\right)=\alpha$. Hence, it is convenient to change the radial coordinate $r_{o}$ to $x=r_{o}-r_{\chi}$, which is centered on the resonant surface $x=0$, so that $\chi^{2}=\left(\mu^{\prime}\left(r_{\chi}\right)\right)^{2} x^{2}$. Equation (17) now becomes

$$
\frac{\partial}{\partial x}\left(\frac{x^{2}}{\left(1+\xi_{x}\right)^{2}}-h(x)\right)=2\left(x^{2} \xi_{\theta \theta}+D_{M} \xi\right)
$$

where

$$
h(x)=\frac{x^{2}}{2 \pi} \oint \frac{d \theta}{\left(1+\xi_{x}\right)^{2}}
$$

and $D_{M}=2 r \beta^{\prime} \kappa^{2}\left(\mu^{\prime}\right)^{-2}$ is the stability parameter of linear theory. Expression (21) is the consequence of the periodicity constraints on the solution of Eq. (20).

The closed system of Eqs. (20) and (21) describes the helical equilibrium associated with a localized interchange mode.

Boundary Conditions While deriving the above equations we have issumed that:

a) each perturbation must be periodic in $\theta_{0}$; and 
b) there is a rational magnetic surface $\mu=n / m$, where the structure of the magnetic field is resonant with the helical structure of the perturbation. If $n / m$ is a simple fraction, then all resonant perturbations must be periodic in $m \theta_{0}$. We can account for this complex periodicity by making the scaling transformation

$$
(\hat{\theta}=m \theta, \ddot{\xi}=\xi / m, \tilde{x}=x / m)
$$

In the limit of 'localized' modes, i.e., $m \gg 1$, the equations are invariant, so that after transformation we retain the same equations but with only simple periodicity in $\check{\theta}$. In the following we assume that this transformation is carried out.

The radial boundary conditions for the internal modes are, as usial, given by:

a) finite value of radial displacement on the magnetic axis, $\xi(0)<\infty$,

b) zero radial displacement at infinity or on the conducting wall, $\xi\left(a_{*}\right)=0$.

For localized modes it is equivalent to $\lim _{x \rightarrow \infty}\left(x^{2} \xi\right)=0$.

\section{Discussion}

1) The linearized version of Eq. (20) is

$$
\frac{\partial}{\partial x}\left(x^{2} \frac{\partial \xi}{\partial x}\right)+x^{2} \xi_{\theta \theta}+D_{M} \xi=0
$$

This is the usual stability problem that results in the well-known $D_{M}<1 / 4$ Mercier criterion. ${ }^{7,8}$

2) Disregarding the right hand side of (20) (it is relatively small in the nonlinear layer) yields

$$
\xi_{x}=\frac{x}{(h(x)+g(\theta))^{1 / 2}}-1 .
$$

Such an expression also appears in Rosenbluth et al. ${ }^{1}$ In this respect the above description of the nonlinear layer is almost equivalent to that used for the $m=1$ mode.

3) The dynamic equations for the low- $m$ modes and the energy functional based on the same principles of derivation have been earlier published in Ref. 3. 


\section{Equilibrium Displacement}

Properties of the Solution The nonlinear equation, derived in the previous section, has the trivial solution $\xi \equiv 0$, which is the axisymmetric initial equilibrium. 'Therefore, if it has other solutions, which is what we are looking for, they are not unique. In fact, there is an infinite number of different solutions, and the first part of this section is devoted to the problem of determining the most interesting one.

Poloidal Wavenumber Assume that there is at least one nontrivial solution of Eq. (20). Then we can make scaling transformations of this function in accordance with Eq. (22), and the result will satisfy the original equation as well as the boundary conditions. Thus we get an infinite set of solutions for every integer $\tilde{m}$. This feature is, of course, conserved for eigenfunctions of the linearized equations, and all of them have the same eigenfrequencies.

If the poloidal wavenumber of the parent helical solution is not the same as the denominator of a simple fraction $\alpha=n / m$ that describes the helicity of the wave, then the parent solution can be upgraded to wavenumber $m$ by the inverse transformations. This is the smallest wavenumber we can obtain without violating the boundary conditions.

If any nontrivial helical solution exists, then there is a whole set of them, with poloidal wavenumbers that are multiples of $m$.

It is easy to see, that the most interesting solution is the one with the lowest poloidal wavenumber. This has the broadest radial extent and the largest radial displacements, according to the features of the transformation in Eq. (22). In what follows, we choose to consider this function only. Note that its characteristic wavenumber $m$ means $\dot{m}=1$ in the normalized equations. 
Radial Symmetry Equation (20) is invariant under two symmetry transformations:

$$
\left[\begin{array}{l}
\theta \rightarrow \theta+\vartheta \\
\left\{\begin{array}{l}
x \rightarrow-x \\
\xi \rightarrow-\xi
\end{array}\right.
\end{array}\right.
$$

Consequently, the most generai form of symmetry that can be imposed on a solution is $\xi(x, \theta)=-\xi(-x, \theta+\vartheta)$. Using it twice in succession leads to $\xi(x, \theta)=\xi(x, \theta+2 \vartheta)$, and for $\tilde{m}=1$. we have $\vartheta^{9}=\ell \pi$ to satisfy the periodicity.

Thus, there are two basic types of symmetry for a helical equilibrium:

$$
\xi(x, \theta)=-\xi(-x, \theta)
$$

which corresponds to the 'tearing parity' mode, and

$$
\xi(x, \theta)=-\xi(-x, \theta+\pi)
$$

which corresponds to the 'ballooning parity' mode.

The linear problem yields the same stability threshold for ballooning and tearing parity perturbations. But for the tearing parity, we already have $\xi(0, \theta) \equiv 0$, so that the interchange of flux tubes cannot take place across the resonant surface. Moreover, the relaxation of the pressure gradient will be much less than that for ballooning parity perturbations, which are, therefore, more interesting. This statement will have its confirmation in the following sections.

Radial Nodes Consider the eigenfunctions of the linearized problem with the same type of symmetry and the same poloidal wavenumber. There are still many of them for any given $D_{M}$ above the instability threshold. They differ in the number of radial nodes in $x$.

To establish the selection principle that is suitable for this kind of degeneracy, we will show that the perturbation with more radial nodes in $\xi^{\prime}(x)$ corresponds to a smaller total displacement on the resonant surface. Hence it corresponds to a smaller relaxation of potential energy and is less interesting. 
Equation (20) has no other parameters besides $D_{M}$, which can be used as an expansion parameter for the description of the nonlinear layer only if $D_{M} \ll 1$, whereas the instability threshold is $D_{M} \geq 1 / 4$. In lieu of an exact solution there are only two options: either to make computer simulations, which are presented in Sec. V, or to derive analytic estimates, based on numerically small factors such as $1 / 4$. These estimates are not asymptotic in any physical parameter, but, nevertheless, they can be based on physical assumptions and they can provide understanding of saturation. To make such estimates is the aim of the following discus! in.

Sheet Currents and Saturation of Interchange It is well known that resonant perturbations of the magnetic field in ideal MHD lead to the formation of sheet currents. ${ }^{1,4}$ In our representation they appear as layers of closely packed flux surfaces, which situation corresponds to $\hat{\xi}_{x}=-1$. Everywhere else $\hat{\xi}_{x}$ must be larger than 1 , since the flux surfaces are initially nested and cannot intersect within ideal MHD.

Let us consider an arbitrary, ideally unstable MHD perturbation in flux-surface related coordinates (Fig. 1). While it is small, it is exponentially growing in amplitude with time. Eventually the $\xi\left(r_{o}\right)$ profile of this perturbation reaches the stage when the $\xi^{\prime}\left(r_{0}\right) \approx-1$ point appears. This corresponds to the formation of a 'sheet current', and the mode cannot grow further without changing its form. Broadening the profile of the perturbation means an increase in the perturbation of the magnetic field and is stabilizing to the mode.

If the mode is just above the linear stability threshold, its amplitude will saturate before the 'sheet current' stage is reached. If there is no other nonlinearity, finding when the $\xi^{\prime}=-1$ stage begins is a simple way to estimate the saturated displacement. Since the sheet current will first appear at the inflection point of the $\xi\left(r_{o}\right)$ profile, we have

$$
\hat{L} \xi=0 ; \quad \xi^{\prime}\left(r_{e}\right)=-1 ; \quad \xi^{\prime \prime}\left(r_{e}\right)=0
$$

These relations determine the position of the inflection point, $r_{e}$, on the eigenfunction of the 
linear equation, $\hat{L} \xi=0$, and hence the amplitude, $\xi$, of the saturated perturbation.

If the mode is far above the threshold for instability, it is necessary to match the external linear solution onto whatever description of the nonlinear layer is available. The geometrically simplest approximation is a 'sheet current' of zero width (or $\xi^{\prime}=-1$ over a finite region of $\left.r_{o}\right)$. Physically this is impossible, since it will cause an infinite perturbation of energy. The real layers are thin, but not infinitely thin. However, this assumption is approximately valid for the task of constructing the $\xi^{\prime}$-continuous perturbation using the linear eigenfunction and the layers.

We wii.' now try to carry out this program for our problem of ideal interchange modes. However, we have to avoid two difficulties:

a) above the instability threshold, the solutions of the linearized equation (23) are oscillatory (as $\xi^{\prime}(x) \propto x^{-3 / 2} \cos \left(\nu \ln \left(x / x_{p}\right)\right)$ for $x \rightarrow 0$ ), so that there are many inflection points;

b) the amplitude of the oscillations in any linear solution increases and becomes infinitely large near the singular point at $x=0$, so that even for small amplitudes the problem seems to be nonlinear.

The cause for these difficulties is the static nature of our analysis. In a real linear stage of instability, the number of nodes is limited and the singularity is removed because of the presence of inertia. Equation (23) is valid only far from the resonant surface, whereas in the inner region it must be modified by either nonlinearity or inertial terms. Effectively, the helical equilibrium is always based on a strongly nonlinear mode, 'far' from the threshold of instability.

Consider a linear eigenfunction growing with time, which has $k$ inflection points $x_{e}>$ $x_{1}>\ldots>x_{k-1}$ at positive $x$. Since the amplitude of the oscillations in $\xi^{\prime}(x)$ is proportional to $x^{-3 / 2}$, the sheet current condition $\xi^{\prime}=-1$ will first be satisfied at the inner inflection point $x_{k-1}$. So that the external perturbation will not be able to grow further. Assuming 
that the inner layer settles down into some nonlinear configuration (see Sec. IV), we arrive at a helical equilibrium with $k-1$ radial nodes in the external region. The widest perturbation with the largest displacement at the matching point corresponds to $k=1$.

Ballooning Parity We now look for a solution that is basically $\tilde{m}=1$, has as few nodes in $x$ as possible, and has ballooning parity, $\xi(x, \theta)=-\xi(-x, \theta+\pi)$. Because of the assumed parity, $\xi(x)$ can have even zero nodes, while $\xi^{\prime \prime}$ has at least one node at positive $x$.

Assume that the perturbation far from the resonant surface is completely $\tilde{m}=1$ : namely, $\xi_{\text {ext }}=\xi_{1}(x) \cos \theta$. This is reasonabie, since in that region the equations are almost linear and the linear solution for the $\tilde{m}=1$ harmonic is the widest in radius, so that all high- $\check{m}$. corrections are concentrated closer to the resonance. As an approximation for the nonlinear internal solution we choose $\xi_{\text {int }}^{\prime}=-1$ over the region $-\delta<x<x_{s}(\theta)$. This is a thin sheet-current layer of variable density.

As a first step, we match displacements at $\theta=0$ and $x=x_{s}(0)$, where the nonlinear layer is widest in $x$ : $\xi_{\text {ext }}\left(x_{s}(0), 0\right)=\xi_{\text {int }}\left(x_{s}(0), 0\right)$. Between the inner boundary of the external solution and the outer boundary of the sheet-current at $\theta \neq 0$, there is now a gap (or overlapping) with undefined magnetic surfaces. We do not need its exact description at the moment, but we must assume that there is no overlapping, so that the outer solution is indeed the outer one for all $\theta$.

As a second step we match the radial derivatives of the displacement at the same point, which is equivalent to balancing radial forces: $\xi_{e x t}^{\prime}\left(x_{s}(0), 0\right)=-1$.

The final step is necessary to define the matching point, $x_{s}(0)$. As discussed earlier, it is logical to match the linear solution onto the sheet current at the minimum of $\xi^{\prime}$ and hence $\xi_{e x t}^{\prime \prime}\left(x_{s}(0), 0\right)=0$ defines $x_{s}(0)$. The resulting curve is shown in Fig. 2.

Let us define $x_{e} \equiv x_{s}(0)$, which is the flux width of the sheet-current layer, and rewrite 
the resulting set of equations as:

$$
\left\{\begin{array}{l}
\xi^{\prime}\left(x_{e}\right) \approx-1 \\
\xi^{\prime \prime}\left(x_{e}\right)=0 \\
x_{e}^{2} \xi^{\prime \prime}\left(x_{e}\right)+2 x_{e} \xi^{\prime}\left(x_{e}\right)+\left(D_{M}-x_{e}^{2}\right) \xi\left(x_{e}\right)=0
\end{array}\right.
$$

Through $x_{e}$ we can estimate another physically significant parameter of the equilibrium configuration, namely, the characteristic displacement of the resonant flux surface, $\xi(0)$ :

$$
\xi(0)=\xi\left(x_{e}\right)+x_{e}=x_{e}\left(1+\frac{2}{D_{M}-x_{e}^{2}}\right)
$$

where $x_{e}$ is the solution of Eq. (26).

In general, solutions of Eqs. (26) and (27) are related to the roots of complex Bessel functions and can be found numerically. An asymptotic evaluation yields the exponential behavior near the threshold of instability:

$$
x_{e} \propto \sqrt{D_{M}} \exp \left(-\frac{\pi}{\left(D_{M}-1 / 4\right)^{1 / 2}}\right) .
$$

These analytic estimates reproduce the $D_{M}$ dependence, as well as the value of the saturated displacement for the ballooning-parity interchange mode. For a given poloidal wavenumber $m$, the above result must be divided by $m$ to obtain the absolute value of displacement.

Tearing Parity For these modes the perturbation must satisfy $\xi(x, \theta)=-\xi(-x, \theta)$, and hence $\xi(0, \theta)=0$. The resonant surface is not displaced from its original position and the sheet-current layer lies along it. This requirement is enough to define the position of the matching point, $x_{e}$, after we go through the first two steps of matching, following the procedure for ballooning parity mode: $\xi(0)=x_{e}+\xi\left(x_{e}\right)=0$. Now

$$
\left\{\begin{array}{l}
\xi^{\prime}\left(x_{e}\right) \approx-1 \\
x_{e}+\xi\left(x_{e}\right)=0 \\
x_{e}^{2} \xi^{\prime \prime}\left(x_{e}\right)+2 x_{e} \xi^{\prime}\left(x_{e}\right)+\left(D_{M}-x_{e}^{2}\right) \xi\left(x_{e}\right)=0
\end{array}\right.
$$


Again, this set of equations can be solved numerically, but the only important thing to know is that the flux width and the characteristic displacement are much smaller than those for the ballooning parity mode:

$$
x_{e_{t}} \approx x_{e_{b}} \exp (-\pi / 2 \nu) \ll x_{e_{b}} .
$$

Here $\nu=\left(D_{M}-1 / 4\right)^{1 / 2}$ measures the extent by which the threshold for linear instability is exceeded.

Discussion In this section we have noted that the nonlinear solution is not unique, and that several different helical equilibria are possible in the system. The most dangerous perturbation, which generates a new equilibrium with the lowest potential energy and largest displacement of flux surfaces, is the ballooning parity perturbation with the lowest poloidal wavenumber consistent with periodicity requirements and without radial nodes in the radial displacement.

We have found an estimate of the saturated displacement for this mode. It is based on the assumption that for a linearly unstable perturbation there is a simple geometric limit for its growth: namely, that the radial derivative of the radial displacement, $\xi^{\prime}$, cannot exceed unity. The reason for this limit is the flux conservation law, which prohibits the intersections of initially nested magnetic surfaces. The estimate is obtained by matching external linearized solution onto the sheet current layer of zero width. The actual current layer has a finite width, and further calculations show that our result for the saturated displacemerit can be larger then the real value by about $20 \%$. A comparison with the numerical model will be given in Sec. $\mathrm{V}$.

The matching procedure and its result are virtually independent of the details of the perturbation structure in the nonlinear region. This means that the source of instability and the cause of saturation are both associated with the external region. Nevertheless, a knowledge of the actual structure of the sheet current and of the whole nonlinear layer is 
important for a physical understanding of reconnection and other dissipation phenomena. This problem is addressed in the next section.

\section{Structure of the Nonlinear Layer}

Hydrostatic Model. To understand the structure of the inner nonlinear layer, we need a simple physical model, which can even involve no magnetic field at all, since the dominant process here is the fluid convection.

Consider a flat horizontal layer of stratified fluid between fixed boundaries in a vertical homogeneous gravitational field. To label the constant density lines, we insert into the fluid thin flexible and elastic horizontal membranes. They will move with the fluid and separate fluid layers of different density, exactly like the flux surfaces do in ideal MHD (Fig. 3a). It is easy to find the 2-D equilibria of this model in the limit of zero elasticity, when it is equivalent to our model of interchange with zero magnetic field.

To reduce the number of possible equilibria that are almost equivalent (having the same density profiles but different distributions of membranes), let us adopt the procedure of selecting only those equilibria that have the smallest length of membranes. This allows for small background elasticity and eliminates thin folds on perturbed membranes. We also have to impose periodicity constraints in the horizontal direction, so that a sequence of permitted waveriumbers is selected.

There are two types of equilibria tha have the lowest potential energy. One is the initial unperturbed equilibrium in a stable case $\nabla \rho_{0} \cdot \vec{g}>0$, and the other is the 'ballooning'-type. equilibrium for $\nabla \rho_{0} \cdot \vec{g}<0$, which is shown in Fig. 3b. The 'tearing'-type solution is also possible, but it can utilize only 1/4 of the available energy (Fig. 3c) and hence is unstable.

The 'ballooning'-type solution in this model can be interpreted as a completed interchange, resulting in a sequence of 'bubbles' on the resonant surface. In a more realistic model with a finite magnetic field, the interchange cannot be completed because of the field 
elasticity; this will be discussed later. In reality the external boundaries of the layer are perturbed by an external solution going as $\approx \xi(0) \cos \theta$. This complication is addressed in the following paragraph.

Equilibrium in a Perturbed Layer Let us consider a thin channel, deformed as $\xi \cos \theta$, so that the width of the channel is $\delta \ll \xi$. The deformation occurred after the channel was filled with stratified fluid, as described in the previous paragraph. After redistribution of fluid, the density gradient returns to its original vertical direction, while possible positions of the membranes are shown in Figs. $4 \mathrm{a}$ and $4 \mathrm{~b}$.

In this model the elastic membranes are equivalent to flux surfaces in a plasma layer. Using this analogy, we interpret configurations in Fig. 4 in terms of the magnetic field and current:

a) In the initially stable case, $\nabla \rho_{o} \cdot \vec{g}>0$, the flux surfaces are compressed together on the inner side of the channel at $\theta=0$ or $\theta=\pi$. At $\theta=\pi / 2$ the direction of magnetic field is horizontal and in the direction of the original unperturbed magnetic field.

b) In the unstable case, $\nabla \rho_{\circ} \cdot \vec{g}<0$, the flux surfaces are compressed together on the outer side of the channel at $\theta=0$ and $\pi$. In between, the direction of the magnetic field inside the channel is horizontal and opposite to the direction of the original magnetic field. The configuration of the middle line ( the 'resonant surface') can be viewed as an incomplete 'bubble'.

A layer of compressed flux surfaces is a representation for a shect current. In the unstable case, the sheet current is generated in the right place to be matched with an external solution. The area with low magnetic field around $\theta=\pi / 2$ resembles an island, since the surrounding flux surfaces go through the $X$-points at $\theta=0$ and $\pi$. Inside this 'island' the heavy fluid is always below the light one, so that in this sense the interchange is again completed. 
Formation of 'Bubbles' Now we intend to show that there is always some region where the purely hydrostatic model explored previously is adequate. Then the question whether the 'bubble' of magnetic field can be formed or whether it is incomplete, as in a thin channel, depends on the relative width of the layer. If the hydrostatic model holds in a region wirer than the external displacement, then the 'bubble' is formed. Otherwise, we get an 'island'like structure with a locally reversed magnetic field.

Let us introduce the characteristic parameter $\beta^{*}$, which is the ratio of the hydrostatic pressure, generated by the stratified fluid in a perturbed channel, to the pressure of the reduced magnetic field (or elastic membranes):

$$
\beta^{*}=4 \pi\left(\rho^{\prime} \cdot \delta\right) g \xi_{\max } / B^{* 2}=D_{M} \xi(0) / \delta
$$

where $\delta$ is the flux width of the layer. Note that $\delta$ enters in the denorninator because $B^{*}=B_{0} b^{*} \propto \delta$.

The physically obvious criterion for validity of the hydrostatic model is $\beta^{*} \gg 1$, which can always be satisfied for sufficiently small $\delta$.

We now have a way to estimate the flux width of the 'island',

$$
x_{i s l a n d}=D_{M} \xi(0)
$$

and, from incompressibility, its width in $\theta$ direction,

$$
\delta \theta_{\text {island }} \approx \pi D_{M}
$$

The flux width of the channel becomes comparable to the external displacement when $D_{M} \geq 1$. At the same time, the $\theta$-width of 'island' is of the order of $\pi$, which means that, the 'bubble' is alrnost closed from above. Thus,

$$
D_{M} \geq 1
$$

is the threshold for the formation of 'bubbles'. 
Discussion Although the preceding study, based on the hydrostatic analogy; is purely qualitative, it allows us to draw several important conclusions.

1) In the new helical equilibrium, associated with the ideal interchange instability, magnetic flux surfaces have folds; i.e., at certain places, the sign of the poloidal component of reduced field is reversed. Also, the assumption $\psi_{r r} \gg \psi_{\theta \theta}$ is not valid in the vicinity of the turning points. Thus, Eq. (20) cannot be expected to reproduce internal 'bubble'-like structures. To check this conclusion, we have numerically solved both Eq. (20) and also the full equation (13), as reported in the next section.

2) The structure of the magnetic field around the 'islands' suggests the possibility of forced reconnection inside of the sheet-current layers. ${ }^{6}$ Then the 'island'-like configuration will be transformed into real magnetic islands. However, the pressure profile is already relaxed inside the nonlinear layer, and hence the instability drive will not change significantly with dissipation. The size of the equilibrium displacement is associated mostly with the external perturbation and will evolve on a much slower time scale.

3) The sign of the mean curvature of magnetic field lines for localized modes in a tokamak is often opposite to that required for interchange instability. ${ }^{9,10}$ This parameter plays the role of local gravity close to the resonant surface. Thus, the field distribution even for the 'stable' case can be adequate for the situation when the instability is driven by nonlocal ballooning or kink terms from outside of the interchange layer.

\section{Numerical Solution}

This section contains a qualitative description of the key points of the numerical methods employed for solving the nonlinear equations (20) and (13) and a discussion of the numerical results. 
Algorithm The algorithm for the numerical solution was based on iteration procedures. To clescribe it, we start by rewriting Eq. (20) in integral form:

$$
\xi(x, \theta)=\int_{\infty}^{\infty}\left(\frac{x}{\sqrt{h(x)+f(\theta)+2 \int_{0}^{x}\left(D_{M} \xi+x^{2} \xi_{\theta \theta}\right) d x}}-1\right) d x .
$$

Here $f(\theta)$ is the integration constant, which must be found from the radial boundary conditions at $-\infty$ or from parity requirements. The use of infinity as the integration boundary can be achieved by means of rlumerical matching onto known solutions of the linearized equation (23).

The iteration sequence is generated by inserting an initial approximation into the right hand side of $\mathrm{Eq}$. (34). The values of $h(x)$ and $f(\theta)$ are found from the requirement that the result satisfies proper boundary conditions:

1) The function $h(x)$ is found from $\theta$-periodicity by the solution of the equation $\oint \xi_{x}^{\prime} d \theta=0$, or

$$
\oint d \theta\left(\frac{x}{\sqrt{h(x)+f(\theta)+2 \int_{0}^{x}\left(D_{M} \xi+x^{2} \xi_{\theta \theta}\right) d x}}-1\right)=0
$$

for every $x$ and any periodic $f$;

2) The function $f(\theta)$ is found from the requirement of enforced parity at $x=0$ : viz., $\xi(0, \theta)=-\xi(0, \pi-\theta)$ (balloonirig) or $\xi(0, \theta)=0$ (tearing). This is achieved by means of an internal iteration cycle in $f$.

The evaluation procedure defines a transiormation within a class of smooth functions $\xi(x, \theta)$, which satisfy our boundary conditions, $\xi_{\text {new }}=\hat{S} \xi_{\text {old }}$. If a stationary point of this transformation, $\xi_{s}=\hat{S} \xi_{s}$, is found, then $\xi_{s}$ is the solution of the initial boundary problem. In fact, by definition of $\hat{S}$, it is the solution of Eq. (34), which itself is equivalent to Eq. (20) coupled with boundary conditions. Thus, although there is no proof that the iteration procedure $\xi_{n}=\hat{S}^{n} \xi_{0}$, with $n=1,2 \ldots \infty$, always approaches a solution, if it does converge to some stationary point, this point is the solution. 
Numerically, giver a reasonable initial approximation, from 3 to 6 iterations in $\xi$ are usually enough to make $\xi$ and $\hat{S} \xi$ equivalent with $1 \%$ accuracy: The reason for this convergence lies in the fact that the right hand side of Eq. (20) is typically small (as $\psi_{\theta \theta} \ll \psi_{x x}$ ), at least in the nonlinear layer. Furthermore, Eq. (34) has such a structure that all cleviations from the real solution are multiplied by this 'smallness' each time' we iterate.

Floating Grid Representation of the perturbation in terms of flux-surface-based coordinates saves us from almost all the inconveniences related to the numerical description of the sheet-current singularity. But this has also a reverse side, causing difficulties in simulating more complex field structures, such as 'folds' and 'bubbles'.

The above algorithm with a simple rectangular grid in $(x, \theta)$ works well only as long as we use the simplified version, Eq. (20), instead of the full equation (13). Full solutions can have 'folds' on the flux surfaces (see Sec. IV), so that several values of $\xi$ correspond to a single $(x, \theta)$ point. This problem can be avoided, and essentially the same iteration procedure retained, by the introduction of new poloidal coordinates.

Equation (13) is rewritten in the Appendix with the use of an orthogonal coordinate system $(x, \phi)$, so that it defines the distance between adjacent flux surfaces along the normal direction. This allows the problem to be reformulated as an integral equation like Eq. (34), except that the path of integration lies along $\phi=$ const, instead of along $\theta=$ const. Also, the iteration cycle can be implemented along the same general lines as in the simplified case. The role of an effective small expansion parameter is assumed by the ratio of the characteristic variation length in $x$ to the variation length along the flux surface. This ratio is less than unity even in the case of fully developed 'bubbles' as in Fig. 4b.

There are two features of this calculation scheme, which need some comment:

a) The real space image of any grid generated in the orthogonal coordinate space is strongly dependent on the $\xi$-function itself, so that the grid points tend to migrate along the 
flux surfaces with iterations;

b) The coordinate lines of constant $\phi$ can be very rapidly diverging in real space for quite regular flux surfaces. Thus, if we place grid points on these lines, they will likely become crowded in one place and very sparsely spaced in another.

These difficulties are resolved by employing an equidistant floating grid, which is only partially related to the coordinate system. The grid points were placed on each flux surface so that the distances between neighboring points are constant. This requires recalculation. of the grid in each step of the radial integrations, but keeps the required number of points low and saves time and worry in the other subprocesses.

The actual calculations were conducted in the region $0<x<x_{\ell}, 0<\theta<\pi$, where $x_{\ell} \sim 1$ is the surface for numerical matching onto the linear region. The grid size is typically $120 \times 60$ points with the density of the $x$ grid increasing toward $x=0$. The calculated field structures, described below, reflect the extrapolation of the data on the region $-x_{\ell}<x<x_{\ell}$, $0<\theta<2 \pi$ according to symmetry and parity requirements.

Field Structures The equilibrium field configurations, calculated for ballooning parity perturbation and $D_{M}=0.6$, are shown in Figs. 5 and 6. The solution in Fig. 5 is based on the simplified equation (20), so that the direction of the field lines within the 'island'-like, low-field structures around $x=0, \theta=\pi / 2$ is incorrect. As shown in Sec. IV, these lines must be always horizontal and in the reversed direction for $D_{M}>1 / 4$. This feature is correctly reproduced by the full solution presented in Fig. 6 .

The visible roughness of the grid representation in Fig. 6a is caused mainly by the disproportionately extended scale on the $x$ axis. The real dimensions of the perturbed layer are shown in Fig. 6b, where grid steps are less visible and one gets a more realistic impression of the $\psi_{\phi \phi} \ll \psi_{x x}$ feature. 
Except for this discrepancy in the description of the internal structure of 'islands', the simplified and full solutions are in good agreement with one another. They are also in very good agreemert with the analytical estimates of the equilibrium displacement, derived in Sec. III.

A comparison of the numerical values with the analytic estimates of the displacement, obtained with the assumption of zero thickness for the sheet current laver, is shown in Fig. 7 . All numerical points for $\xi_{0} \equiv \xi(0)$ are slightly below the solid curve, defined by $\mathrm{Eq}$. (26). This difference is, obviously, the real radial width of the current layer. It is well within $20 \%$ of the total displacernent, which is very good agreement, considering the nature of the assumptions.

Figure 8 shows the equilibrium configuration, which corresponds to the locally stable system $\left(D_{M}=-0.5\right)$ with an externally perturbed boundary. The idealized hydrostatic configuration for such a case is shown in Fig. 4a. The field structure again exhibits intermittent 'islands', with a horizontal field inside, and 'sheet'-current layers.

Discussion We have been able to find numerical solutions of Eqs. (13) and (20) by utilizing the same small parameter that was used for the analytical reduction of the problem, although in a somewhat different way. However, the mathematical validity of that iterative approach has yet to be tested.

The numerical results are in good agreement with the predictions of simple models (Sec. III,IV) based on a physical understanding of the problem.

The estimates of the saturated displacement from Sec. III have relatively small deviations from the numerical results. Therefore, the approach that was used in their derivation, can also be used for other problems as well. 


\section{Ballooning Modes}

Relationship to Interchange Modes The closest tokamak analog to interchange modes are the localized Mercier modes. ${ }^{9,10}$ The cylindrical and toroidal cases differ mainly in that the curvature of the magnetic field in a tokamak varies along the field line, and exact helical symmetry for the equilibrium is impossible. However, the usual weak-toroidicity approximation generates a perturbation method that describes all toroidal corrections to the mode as two (or more for higher-order methods) satellite poloidal harmonics. These satellites are equivalent to a small amplitude modulation imposed on the original cylindrical solution. The same physical idea manifests itself as the averaging technique widely used for solving the ballooning equations.

It would be very interesting to check whether this averaging of the toroidal variation of the field curvature can be carried out before linearizing the MHD equations. Then the theory of cylindrical interchange described above would be valid for Mercier modes as well (with a toroidally modified stability pararneter, $D_{M t}=D_{M c}\left(1-q^{2}\right)$, of course). Lacking an explicit proof at the moment, we can provide only qualitative arguments to support this hypothesis:

a) the description of the interchange in the external region is linear, so that all linear techniques can be implemented there;

b) in the internal nonlinear layer the characteristic magnetic energy associated with nonhelical perturbations (3-D effects) is typically $\left(B^{*}(1) / B^{*}(x)\right)^{2} \sim 1 / x_{e}^{2}$ times larger than that related to helical movements. Since $x_{e}$ is very srnall, at least close to the instability threshold, all 3-D effects can be calculated perturbatively.

If we accept this argument, then the toroidal Mercier modes are equivalent to cylindrical localized modes with a modified mean curvature parameter $D_{M t}=D_{M c}\left(1-q^{2}\right)$. With the usual monotonically decreasing pressure profiles and $\epsilon \beta_{p}<1$, such modes are stable since $D_{M}<0$ everywhere, except, perhaps, within the $q(r)=1$ surface. $^{10}$ This feature makes 
nonlocal ballooning modes very important for finite- $\beta$ stability theory.

Ballooning modes can have three main sources of free energy that drives them unstable:

1) the mean curvature of field lines and the pressure gradient, the same as for "ucalized modes;

2) the enhanced utilization of variations in the field curvature, which is related to large radial width of each individual helical perturbation;

3) the interaction of several different helical perturbations shifted in radius (this contribution is the only one that is dependent on the Bloch shift and accounts for the formation of the 'radial structure'11).

We can incorporate in 2-D equations the first effect only, while the nonlocal terms are inherently 3-D and typically very hard to find even in the linear theory. This difficulty was also encountered in the resistive theory of ballooning modes, so that one of the tricks developed there is useful for our purposes.

As in the theory of resistive ballooning modes, ${ }^{12}$ it is possible to assume that the influence of all driving effects outside of the interchange layer can be described by the single parameter $\Delta^{\prime}$. Physically it is equivalent to the statement that ballooning effects can be described as a modification of the boundary conditions for the interchange layer problem. With this assumption, the estimate for the amplitude of the nonlinearly saturated ballooning mode can be obtained. That approach will be valid as long as the $\Delta^{\prime}$-defined boundaries do not interfere with the internal nonlinearity, i.e., the nonlinear layer must be contained within the interchange layer.

$\Delta^{\prime}$-Driven Layer Let us assume the interchange mode itself to be stable, $D_{M}<1 / 4$, and the interchange to be driven from the outside by a $\Delta^{\prime}$-like contribution from the external region. Then the asymptotic solutions for $x \rightarrow 0$ behave like

$$
\xi \propto x^{\alpha_{1}}+\Delta_{b} x^{\alpha_{2}}, \quad\left(\Delta_{b}=1 / \Delta^{\prime}\right)
$$


Here we defined

$$
\alpha_{1,2}=-\frac{1}{2} \pm\left(\frac{1}{4}-D_{M}\right)^{1 / 2}
$$

The ideal ballooning stability threshold is $\Delta_{b}=0$, and we will consider only the unstable case, $\Delta_{b}<0$.

If the largest root of Eq. (37) is $\alpha_{1}<0$, then the mode has the structure shown in Fig. 9. The linear solution has one node in radius and one inflection point, so that $\mathrm{Eq}$. (26) of $^{\mathfrak{b}}$ Sec. III is justified. But in the present case we can use the asymptotic formulae to find the position of the inflection point explicitly:

$$
\xi^{\prime \prime} \propto \alpha_{1}\left(\alpha_{1}-1\right) x^{\alpha_{1}-2}+\alpha_{2}\left(\alpha_{2}-1\right) \Delta_{b} x^{\alpha_{2}-2}=0
$$

Substituting expressions (37) for $\alpha_{1,2}$ we obtain:

$$
x_{b}=\left(\frac{1-D_{M}+\left(1-4 D_{M}\right)^{1 / 2}}{1-D_{M}-\left(1-4 D_{M}\right)^{1 / 2}}\left(-\Delta_{b}\right)\right)^{\left(1-4 D_{M}\right)^{-1 / 2}}
$$

and

$$
\xi_{b}(0)=\xi\left(x_{b}\right)+x_{b}=x_{b}\left(1+\frac{2}{D_{M}-x_{b}^{2}}\right) .
$$

These two equations govern the saturation of ideal ballooning modes in a tokamak. Since the internal description is the same as in a cylinder, all properties of the configuration discussed in Sec. III-V still hold and the accuracy of the above analytic estimate is the same as shown in Fig. 7 for interchange modes.

This analysis is valid for $1 / 4>D_{M}>0$. Otherwise, $\alpha_{1}>0$ and the linearized solution has no inflection points within the interchange layer. This feature of solution means that the sheet-current layer is first generated in the external ballooning region, and we lack the capability to describe it. The internal layer then looks like the one shown in Fig. 8.

Discussion We have found an estimate for the amplitude of the nonlinear saturation of ballooning modes in tokamak. The physical foundations of it are as follows: if the contribution to the potential energy of the mode from the mean curvalure un the resônanint suinface 
is destabilizing, $D_{M}>0$, then the sheet-current induced internal modifications of the perturbation profile can make it stable. Otherwise, for $D_{M}<0$, the dependence of stability on the details of the perturbation profile inside the interchange layer is weak, and the mode will grow until the nonlinear layer becomes wide enough to modify the ballooning effects. Under typical tokamak conditions, the sign of $D_{M}$ is negative.

\section{Conclusion}

Summary The saturation of interchange causes the generation of sheet currents and island. like structures on and around the resonant flux surface. These can be simulated with the hydrostatic model, described in Sec. IV. The almost closed 'bubbles'4 are possible only at large pressure gradients (or low shear), $D_{M} \gg 1$, but the 'folds' on magnetic surfaces are the necessary elements of equilibrium for all $D_{M}>0$.

The characteristic size of the saturated displacement is proportional to the position of

the inflection point in the linear eigenfunction $\xi \propto \sqrt{D_{M}} \exp \left(-\pi\left(D_{M}-1 / 4\right)^{-1 / 2}\right)$. This heuristic dependence is supported by numerical solutions.

The saturation of ballooning modes in a tokamak is analogous to that of the cylindrical interchange if $D_{M}>0$. Otherwise, dimensions of the nonlinear structure are larger then the size of the layer where the interchange approximation is valid.

Problems There is still a number of unresolved problems related to nonlinear interchange and ballooning modes. For example, the mathematical justification for the apparently accurate heuristic formula for the saturated displacement is not convincing and no analytic estimate of its probable error is given. The 'bubble' limit with $D_{M}>1$ is not investigated in detail, while it can be of interest for low-shear discharges.

The approach developed in this paper is also in need of some generalization:

a) We need to consider resistive fluids, to account for the reconnection, which can change 
the topology of the field lines. Heat conductivity and diffusion, which are able to change the local value of $D_{M}$, also should be examined.

b) In order to account for the interaction of two or more saturated structures with different helicities, we need to consider 3-D configurations. Related problems include the saturation of toroidal ballooning modes with $D_{M}<0$, and the possible consequences of the simultaneous excitation of several modes.

Acknowledgments The author wishes to express his gratitude to Dr. O.P.Pogutse of Kurchatov Institute for initiating this research program, and to Drs. A. Y. Aydemir and J. W. Van Dam of IFS for important discussions and assistance in preparing this paper.

This work was supported in part by the U. S. Department of Energy under grant DE-FGO5-80ET-53088. 


\section{Appendix. Equations In Orthogonal Coordinates}

Here we will derive a new form for Eq. (13) in terms of orthogonal coordinates $\left(r_{\circ}, \phi\right)$; where $r_{0}$ still labels the magnetic surfaces throughout the motion, and the definition of $\phi$ follows from orthogonality:

$$
\frac{\partial \phi}{\partial r} \frac{\partial r_{o}}{\partial r}+\frac{1}{r^{2}} \frac{\partial \phi}{\partial \theta} \frac{\partial r_{o}}{\partial \theta}=0
$$

or

$$
r_{r} r_{\phi}+r^{2} \theta_{r} \theta_{\phi}=0
$$

Here subscripts denote partial derivatives in $\left(r_{o}, \phi\right)$ space.

The condition of orthogonality is not enough to provide a unique definition for $\phi$. We have to describe its relation to $\theta$ or $\theta_{0}$ on some one flux surface as well. This choice will be discussed later.

The incompressibility of motion, Eq. (8), in these new coordinates can be expressed as

$$
r_{r} \theta_{\phi}-r_{\phi} \theta_{r}=\frac{r_{0}}{r} \sigma_{\phi}
$$

where $\sigma_{\phi} \equiv \partial \theta_{o} / \partial \phi$ is the Jacobian.

Using expressions (A2) and (A3) it is now possible to rewrite Eq. (13) in $\left(r_{o}, \phi\right)$ coordinates. The aim is, as in Sec. II, to get an expression like

$$
\frac{\partial}{\partial r_{o}} U-H=V
$$

where $U$ and $V$ are differential expressions, but $V$ contains no derivatives in $r_{0}$. (at least in the numerator). Then the right hand side will be much smaller than the left hand side for interchange-like perturbations, and we can implement the iteration procedures of Sec. V.

The equation can be put in such a form if we multiply Eq. (13) by $r^{2}$ and rearrange terms with the help of (A2), (A3);

$$
\frac{1}{2} \frac{\partial}{\partial r_{o}}\left(r^{2} \chi^{2} \Lambda^{-2}\right)-r^{2} H\left(r_{o}\right)=\chi^{2} R-\beta^{\prime} \kappa^{2} r^{2}\left(r^{2}-r_{o}^{2}\right)
$$


Here $A \equiv \sigma_{\phi} /\left(r_{\phi}^{2}+r^{2} \theta_{\phi}^{2}\right)^{1 / 2}$ and has a simple geometrical meaning: it is the differential distance between lines of constant $r_{o}$ along the normal $\phi=$ const. The expression for $R$ is rather complicated, but has no singularities:

$$
R=\frac{r_{o} r^{2}}{\sigma_{\phi}} \frac{\left(r \theta_{\phi} r_{\phi \phi}-r_{\phi}\left(r \theta_{\phi}\right)_{\phi}\right)}{r_{\phi}^{2}+\left(r \theta_{\phi}\right)^{2}}
$$

The $\phi$ coordinate can be normalized on some arbitrary function of $\theta_{0}$, which will then define $\sigma_{\phi}$. Since it is arbitrary, we choose several different functions to use in different portions of the volume. On each boundary between those portions it becomes necessary to recalculate the coordinates, but the inconvenience of doing so is compensated by some advantages, as pointed out in Sec. $V$. 


\section{References}

${ }^{1}$ M. N. Rosenbluth, R. Y. Lagazian and P. H. Rutherford, Phys. Fluids v.16, p.1894 (1973)

${ }^{2}$ Avinash, R. J. Hastie, and J. B. Taylor, Phys.Rev.Lett., 59, 2647 (1987)

${ }^{3}$ A. D. Beklemishev and O. P. Pogutse, Sov.J.Plasma Phys 15, 375 (1989)

4 B. B. Kadomtsev and O. P. Pogutse, Sov. Phys. JETP 38, 283 (1974).

${ }^{5}$ J. F.Drake and T. M. Antonsen,Jr., Phys.Fluids, 27, 898 (1984).

${ }^{6}$ T. S.Hahm and R. M.Kulsrud, Phys.Fluids, 28, 2412 (1985).

${ }^{\top}$ B. Suydam, in Proc. 2-nd U.N. Intern. Conf. P.U.A.E., (Geneva, 1958), Vol.31, p.157.

${ }^{8}$ B. B. Kadomtsev, Hydromagnetic Stability of Plasma, in Reviews of Plasma Physics Ed. M. A. Leontovich, (Consultants Bureau, New York 1966) Vol.2, p.179.

${ }^{9}$ C. Mercier, Nucl.Fusion, 1, 47 (1960).

${ }^{10}$ V. D. Shafranov and E. I. Yurchenko, Sov. Phys. JETP, 53, 1157 (1967); A. A. Ware and F. A. Haas, Phys.Fluids, 9, 956 (1966).

${ }^{11}$ J. W. Connor, R. J. Hastie, J. B. Taylor, Proc. Royal Society, London A 365, 1 (1978).

${ }^{12}$ H. R. Strauss, Phys.Fluids, 24, 2004 (1981). 


\section{Figures}

FIG. 1.Linear growth of the radial displacement in time, which is limited by the generation of a singular current at the inflection point of the eigenfunction.

FIG. 2.Matching scheme for ballooning parity perturbation: (a), slightly overestimates the displacement as compared to the more realistic form (b) with finite thickness of the current layer.

FIG. 3.Hydrostatic model for the internal nonlinear layer of the helical equilibrium $\left(D_{M} \gg 1\right)$. Flat layer with stable density gradient. (a), formation of 'bubbles' in the unstable case (b), partial interchange with enforced 'tearing' parity (c). Constant density lines are rounded at corners to show direction of turns.

FJG. 4.Hydrostatic model for the internal nonlinear layer of the helical equilibrium $\left(D_{M}<1\right)$. Perturbed layer boundaries cause redistribution of constant density lines in initially stable (a) and unstable (b) cases. Density lines correspond to cross sections of flux surfaces, so that details of the configuration may be interpreted as sheet currents and 'islands'.

FIG. 5.Flux surfaces in the new helical equilibrium as given by numerical solution of the reduced Eq. (20) with $D_{M}=0.6$. Initial position of these surfaces was horizontal and equidistant.

FIG. 6. The new helical equilibrium as given by numerical solution of the full Eq. (13) in the local approximation. $D_{M}=0.6$. The same configuration, but in actual proportions, is shown below.

FIG. 7.Comparison of the saturated displacement $\xi(0)$ for the interchange instability as given by numerical solution (dots) and by analytic estimate (upper curve). The difference between the position of the dots and the curve represents the real width of the sheet current layer. The lower curve shows the flux width of this layer, $x_{e}$, which is the position 
of the matching point in the analytic theory.

FIG. 8. Internally stable layer $\left(D_{M}=-0.5\right)$ driven by perturbed boundaries. The configuration resembles that of Fig.4a and may be similar to real structures produced by nonlocal ballooning instability.

FIG. 9. Asymptotic $(x \rightarrow 0)$ torm of a linear eigenfunction for the ballooning instability with $0<D_{M}<1 / 4$. 


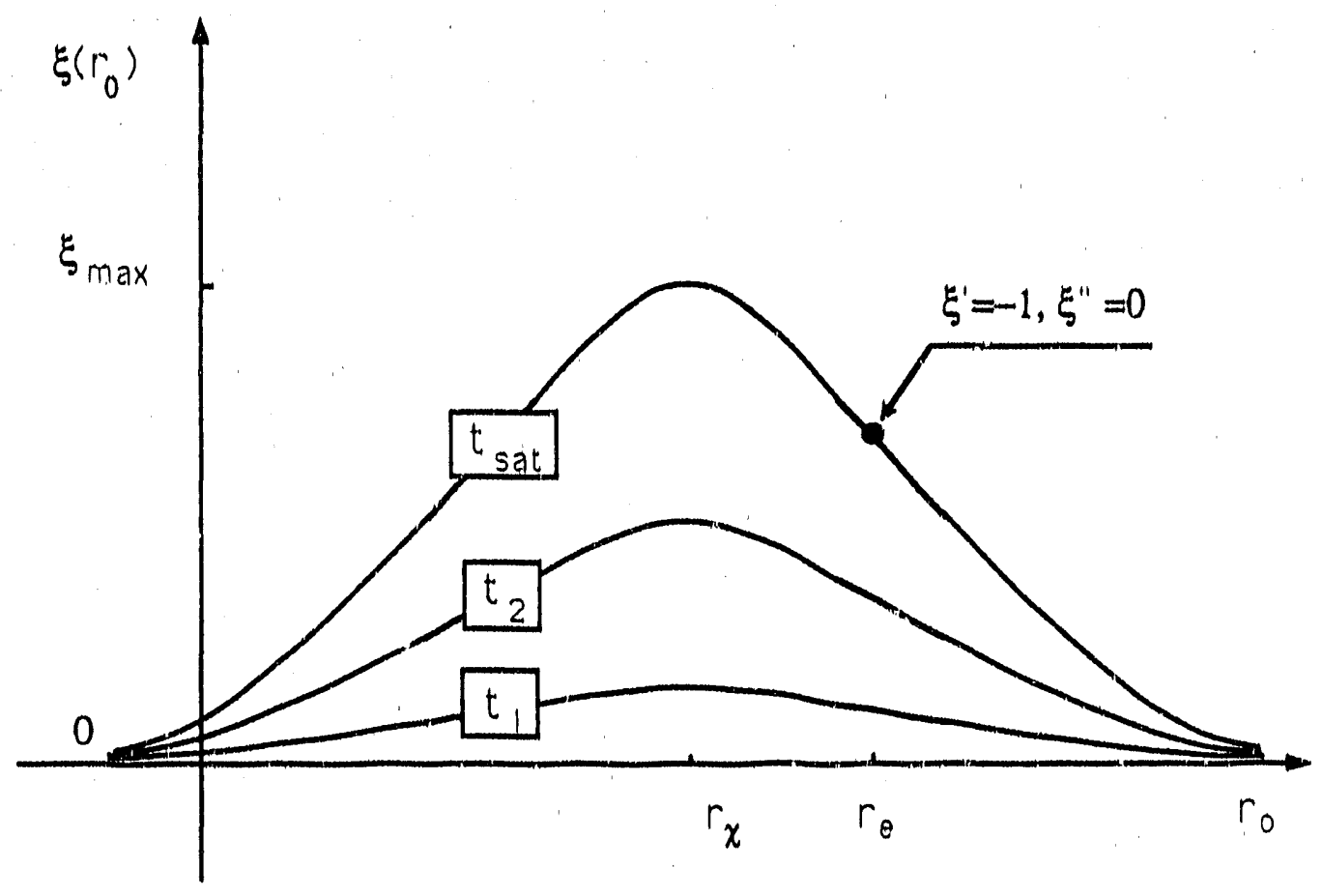

$F|G$, 


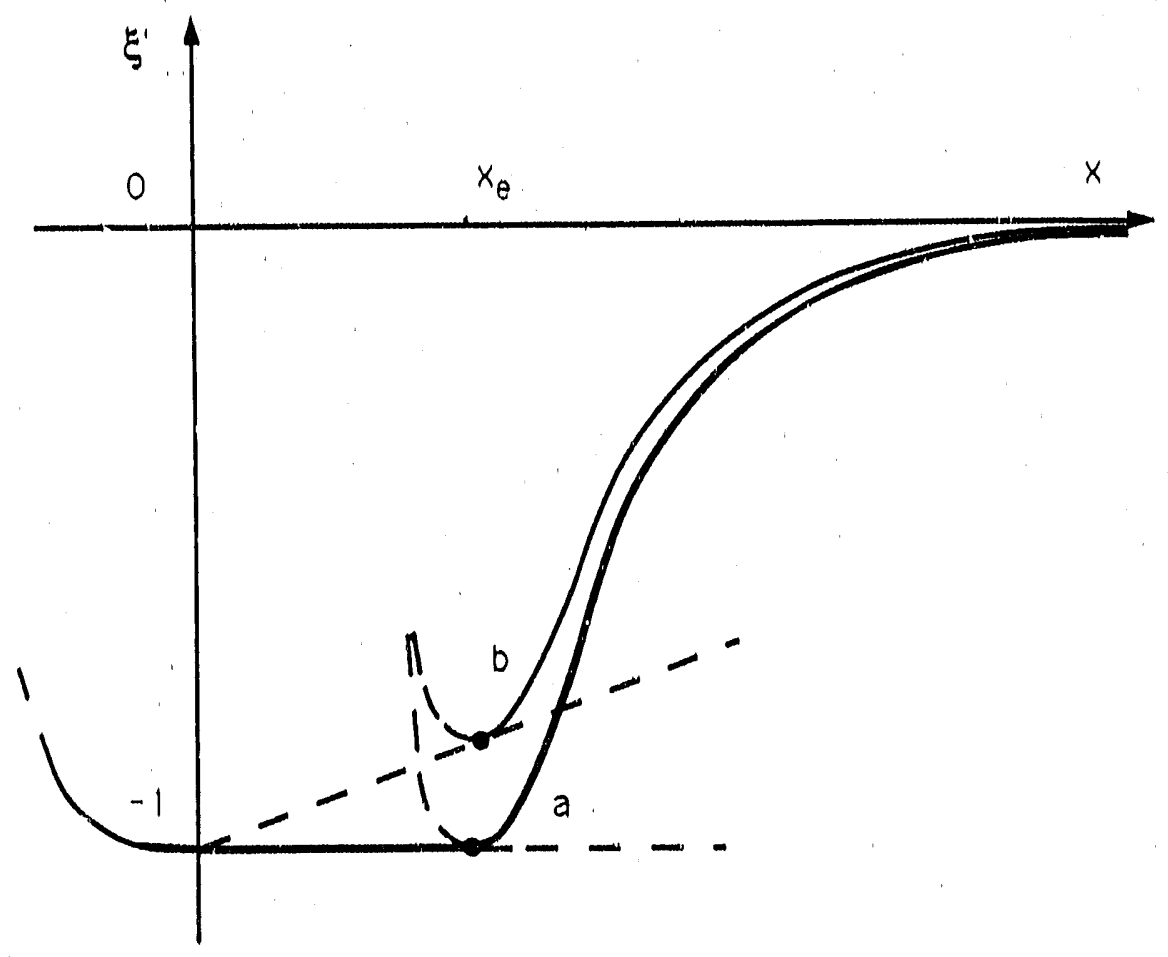

FIG. 2 


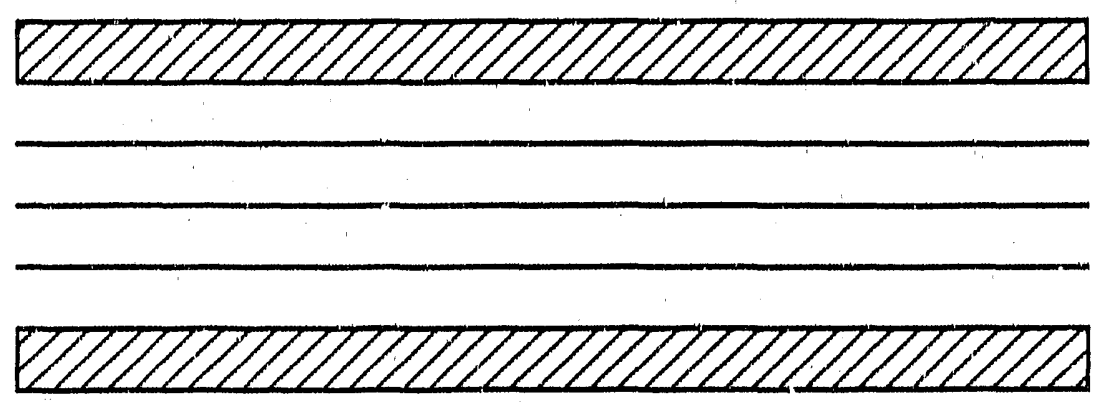

FIG. $3 a$

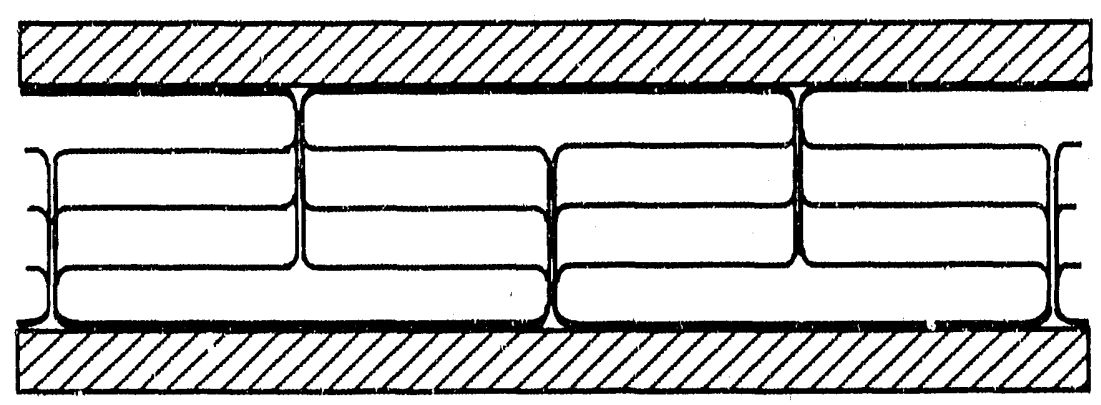

FIG. 3b

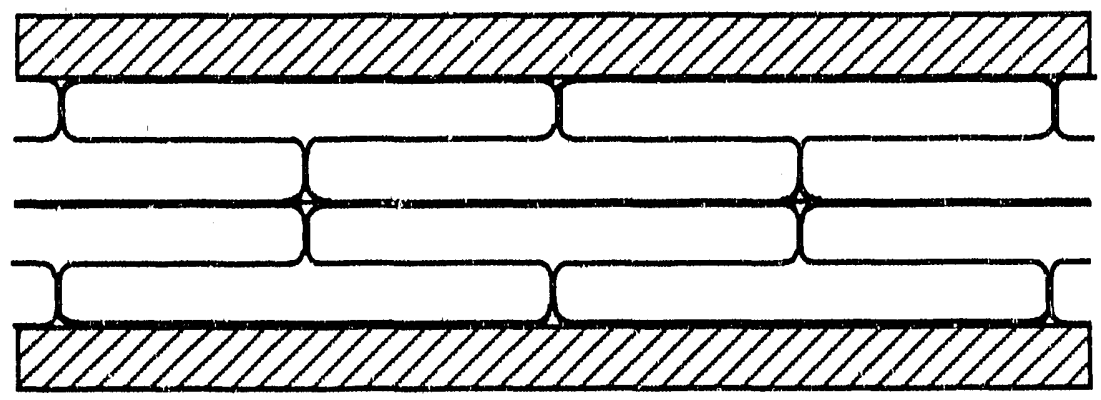

FIG. 3C 


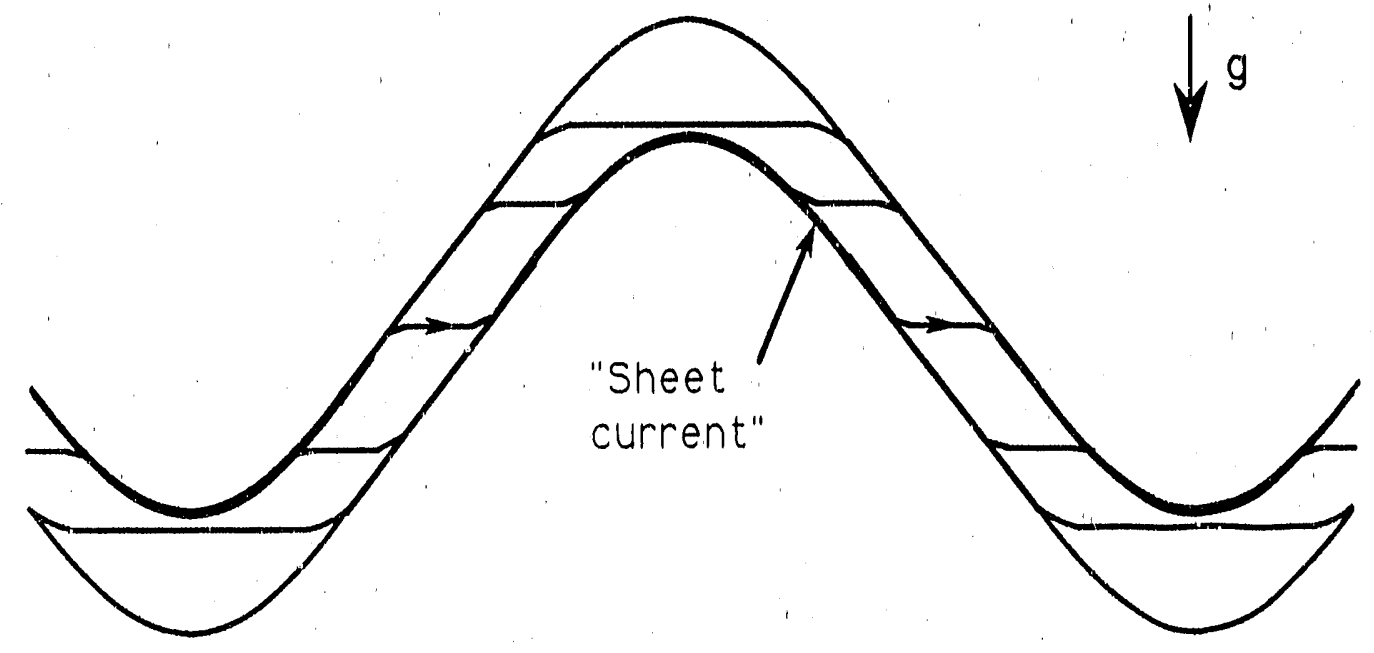

FIG. $4 a$

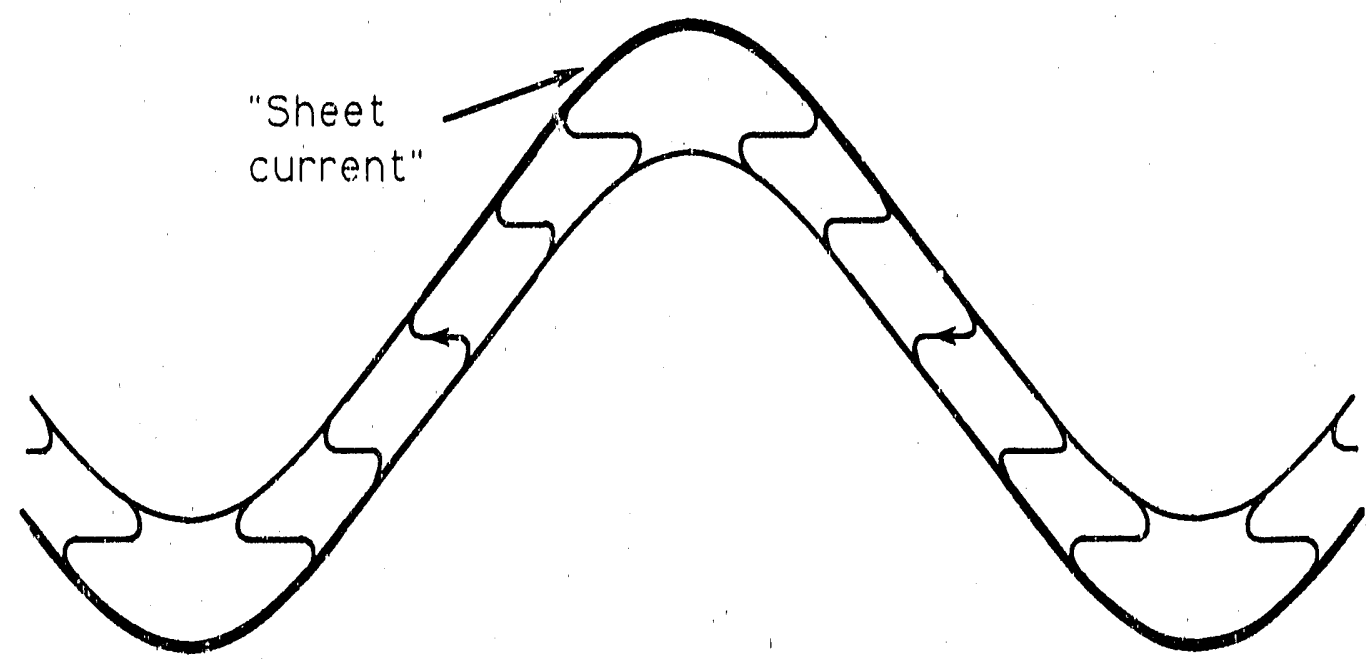

FIG. $4 b$ 


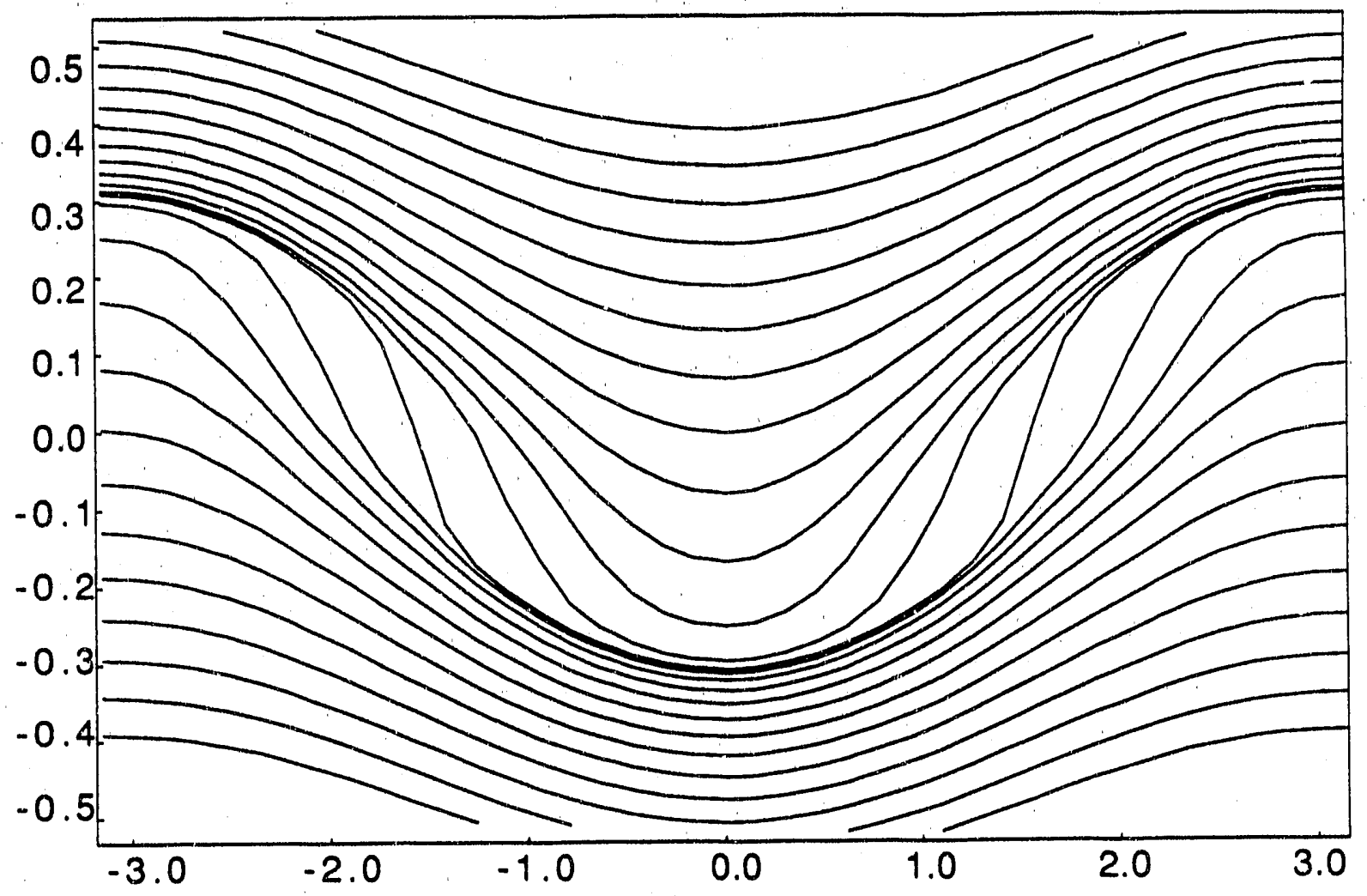

FIG. 5 

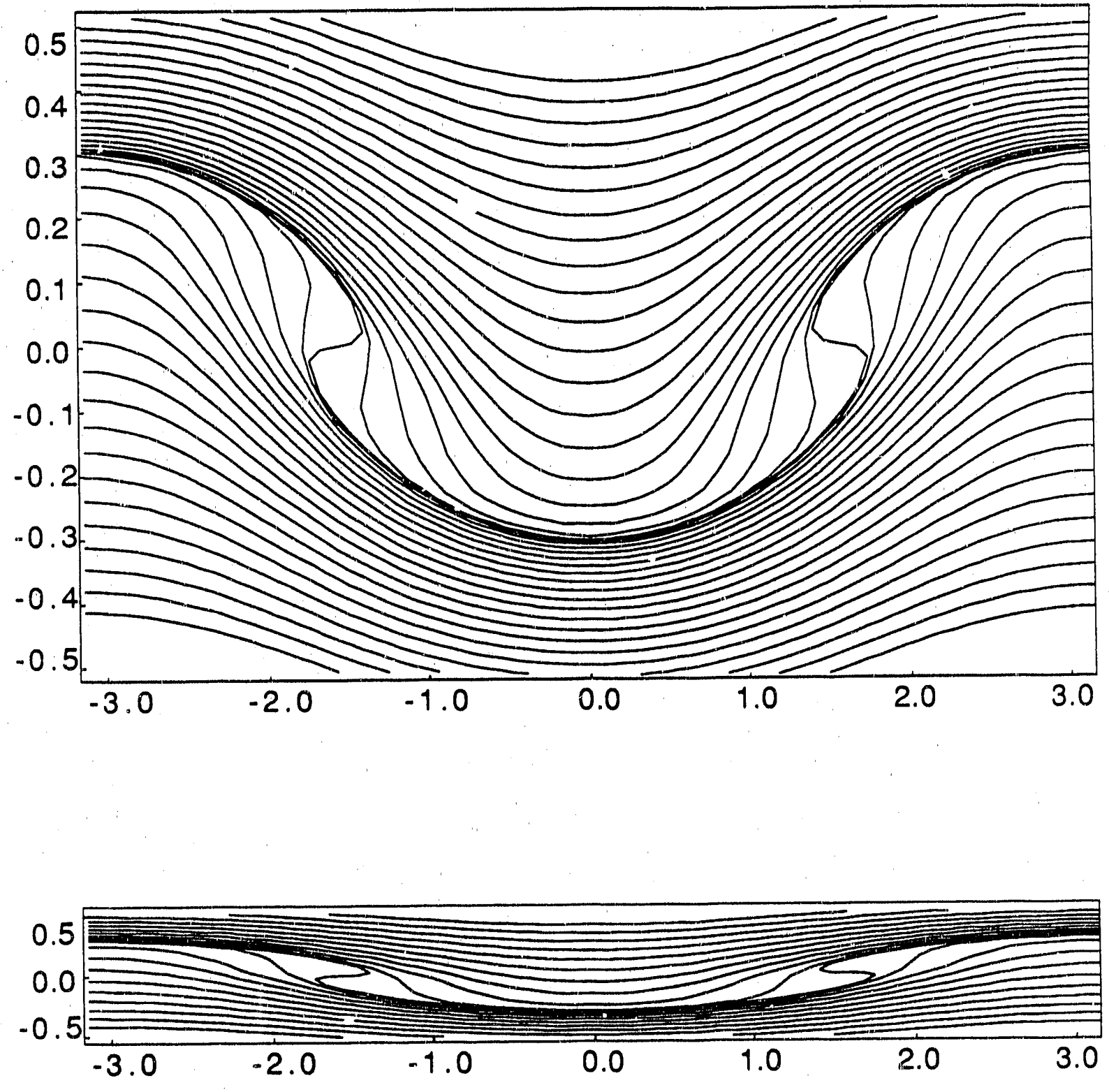

FIG. 6 


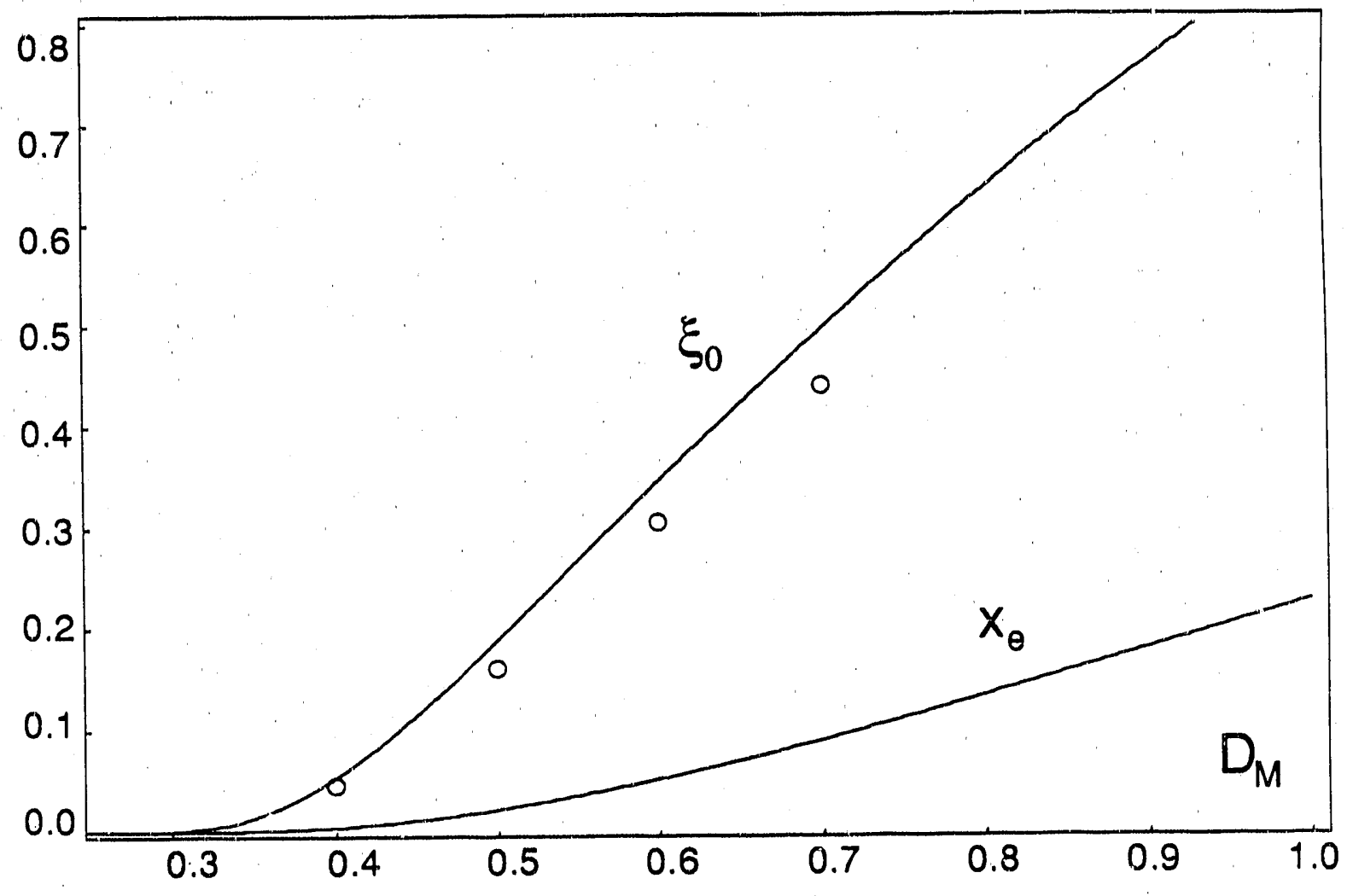

FIG. 7 


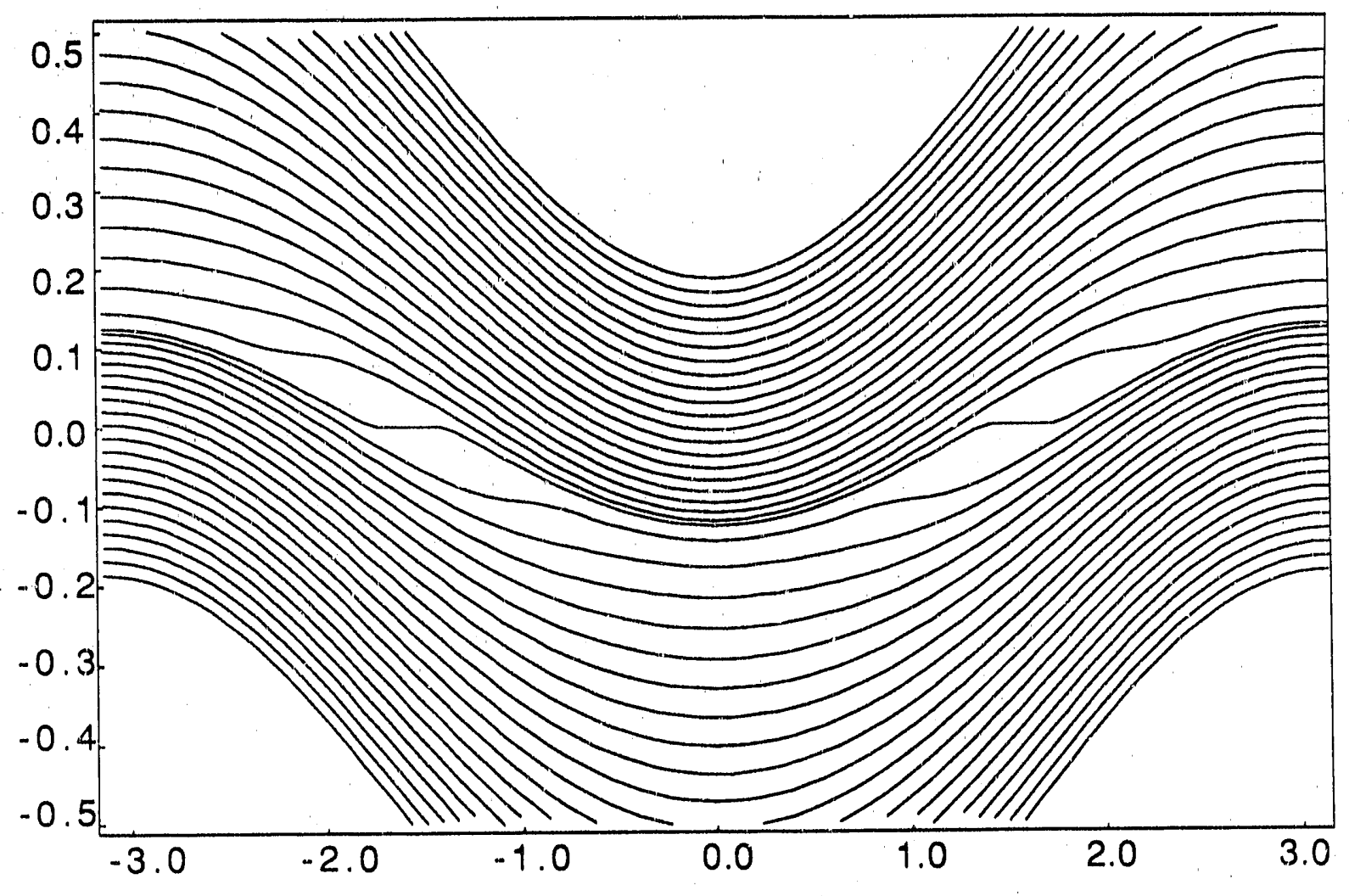

FIG. 8 


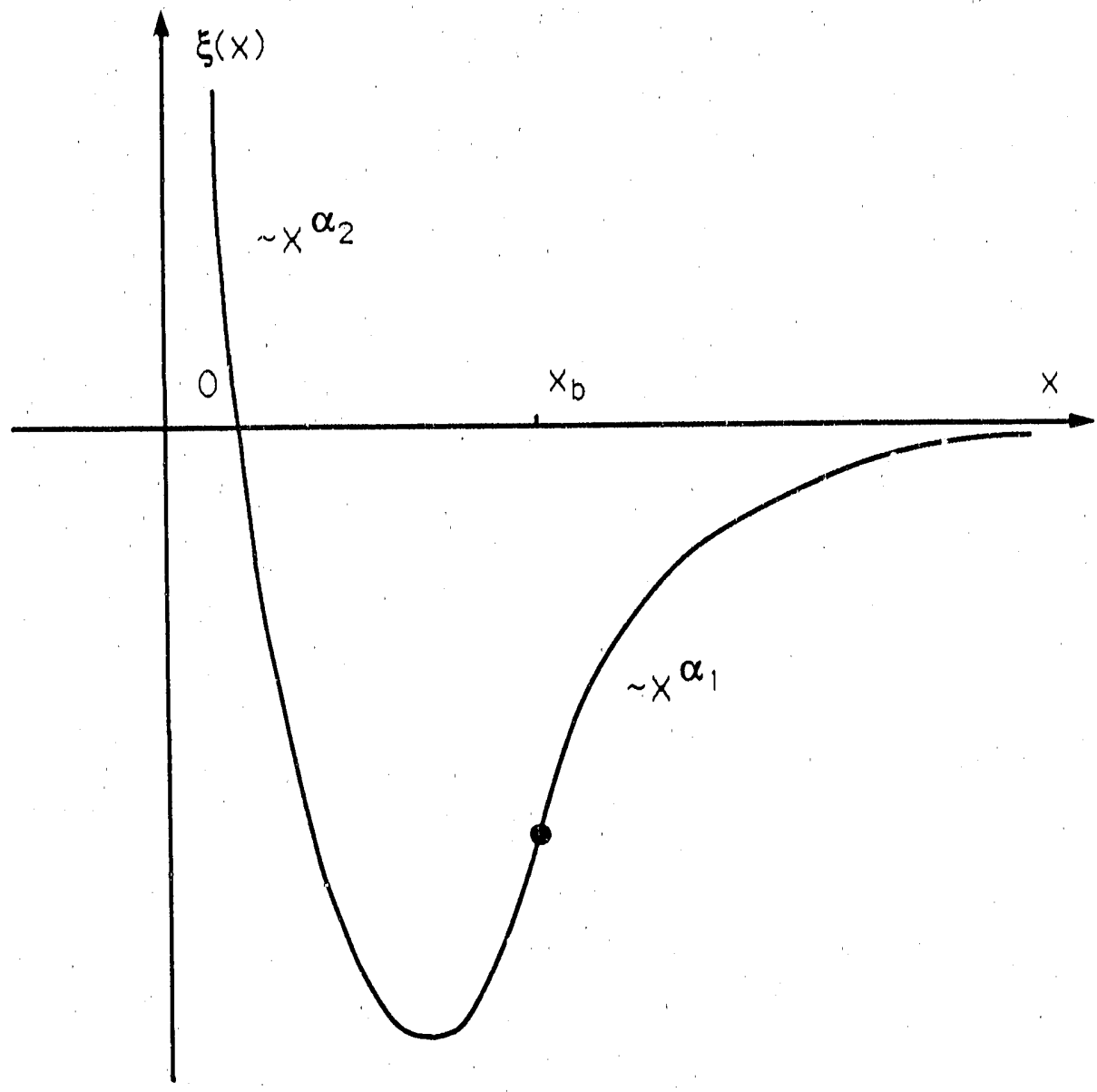

$F \mid G .9$ 

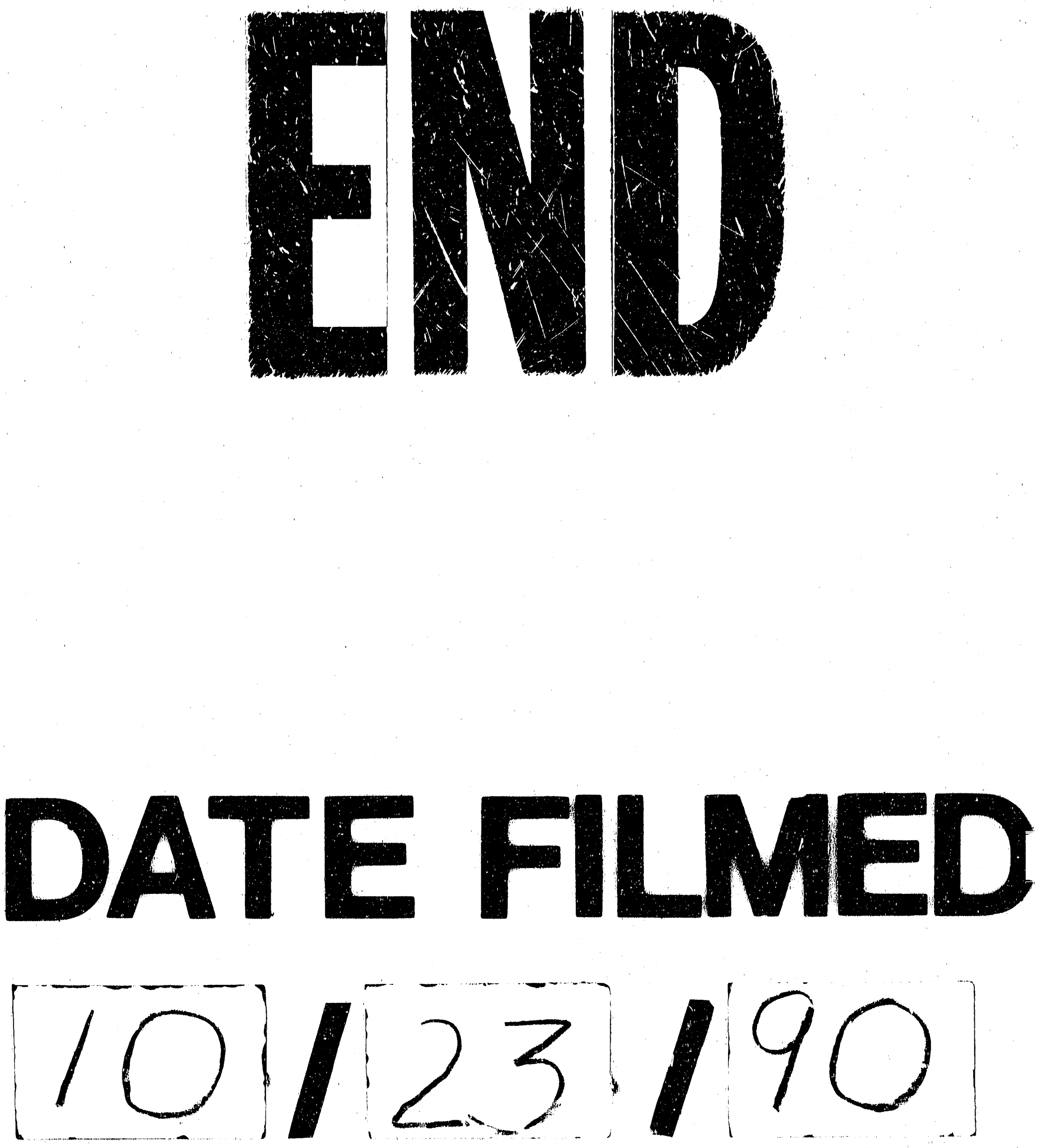

1 
